\title{
Model identification in computational stochastic dynamics using experimental modal data
}

\author{
A. Batou*a, C. Soize ${ }^{\mathrm{a}}$, S. Audebert ${ }^{\mathrm{b}}$ \\ ${ }^{a}$ Université Paris-Est, Laboratoire Modélisation et Simulation Multi Echelle, \\ MSME UMR 8208 CNRS, 5 bd Descartes, 77454 Marne-la-Vallée, France \\ ${ }^{b}$ Électricité de France Lab, Acoustics and Mechanical Analyses Department, \\ 1 avenue du Général de Gaulle, 92141 Clamart Cedex, France
}

\begin{abstract}
This paper deals with the identification of a stochastic computational model using experimental eigenfrequencies and mode shapes. In presence of randomness, it is difficult to construct a one-to-one correspondence between the results provided by the stochastic computational model and the experimental data because of the random modes crossing and veering phenomena that may occurs from one realization to another one. In this paper, this correspondence is constructed by introducing an adapted transformation for the computed modal quantities. Then the transformed computed modal quantities can be compared with the experimental data in order to identify the parameters of the stochastic computational model. The methodology is applied to a booster pump of thermal units for which experimental modal data have been measured on several sites.
\end{abstract}

Keywords: Structural dynamics, Model identification, Computational stochastic dynamics, Mode crossing, Experimental modal analysis

\section{Introduction}

In industrial context, the quantification of the confidence in computational models must be established so that they can be used either in design purpose or in expertise purpose. A given dynamical system can operate at design conditions, at off-design conditions and at failure-mode conditions

\footnotetext{
*Corresponding author

Email address: anas.batou@univ-paris-est.fr (A. Batou*)
} 
that apply in accident scenarios. An adapted way to take into account their generic characteristics and their capability to reproduce the behavior of the whole family of nominally-identical structures is to consider no more deterministic but stochastic computational models (SCM), using experimental data. The objective of this paper consists in identifying a SCM using some natural frequencies and the associated mass-normalized mode shapes, measured for a family of structures.

The identification or updating methods of deterministic computational dynamic models using modal data have been intensively studied during the last four decades. Efficient methods have been proposed (see for instance $[31,51,20])$ and are now commonly used in industry. There are two main types of methods: the global methods (see for instance [3, 21, 41, 54]) which consist in directly modifying the stiffness and mass matrices and the local methods which consists in updating some physical parameters (see for instance $[9,14])$. The latter method can be described in three steps: (1) The first one consists in constructing a nominal computational model (NCM) for which the parameters are set to nominal values. These parameters can be related to material properties (Young's modulus, mass density, and so on), geometry (CAD, thickness, area moments of inertia, and so on) and boundary conditions. (2) The second step consists in performing a sensitivity analysis of the quantities of interest with respect to the parameters in order to select the most-sensitive parameters which have to be updated (see for instance $[6,15])$. (3) The third step consists in updating the most-sensitive parameters using experimental data. In general, this step is carried out by reducing a "distance" between the experimental modal data (eigenvalue, mode shape, modal mass, modal damping) and the corresponding computed quantities using the NCM.

We consider the random context for which the available experimental data are related to a family of several experimental configurations of a given dynamical structure. The observed variability between the experimental configurations of this family is induced (1) by the uncontrolled differences that can appear during the manufacturing process (manufacturing tolerances) and during the life cycle of the structure (natural damage, incidents, etc) and (2) by some slight differences which are controlled and are related, for instance, to the boundary conditions, the embedded equipments, etc. These two types of variability induce differences for the data measured on two configurations of the given dynamical structure. It should be noted that the measurement 
errors can also yield fluctuations in the measured data. This last source of variability induced by the experimental process is not addressed in this paper because it is assumed to be of second-order with respect to the other sources of variability for the application which is presented.

In such a random context, we have to construct a stochastic computational model (SCM) for which two sources of uncertainties have to be taken into account (see for instance [48]): (1) the uncertainties relative to some model parameters of the NCM and (2) the modeling errors. The first one includes both the uncertainties induced by the above-mentioned observed variability (also called aleatory uncertainty in the literature) and the uncertainties induced by the lack of knowledge related to some uncertain parameters of the SCM (also called epistemic uncertainty in the literature). With respect to the computational model, this first source of uncertainty yields the model-parameter uncertainties while the uncertainties induced by the modeling errors yield the model uncertainties. The stochastic computational model which is constructed with these two sources of uncertainties (and with additional input and output noises if measurement errors are significant) must have the capability of representing the variability of all the measured configurations (as explained above).

In this paper, the uncertainties are taken into account using a probabilistic approach and then the SCM is constructed including both the modelparameter uncertainties and the model uncertainties in a separate way (using the generalized probabilistic approach of uncertainties proposed in $[47,48]$ ). Usually, a SCM is controlled by a set of hyperparameters such as mean values, coefficients of variation, and so on. These hyperparameters have to be identified using experimental data and realizations of the SCM. Several types of observation can be used in order to perform such an identification and the choice depends on the quantities of interest for the developed SCM (see for instance, [34, 35, 19, 22, 23] for model-parameter uncertainties, and $[45,46,12,13,5,2,49]$ for both model-parameter uncertainties and model uncertainties). For example, if the computational model is devoted to the prediction of responses in the low-frequency band of analysis and if the resonances are relatively well-separated, then the modal quantities (eigenfrequencies, mode shapes) are suitable observations. In a random context and for the low-frequency dynamical analysis, the eigenfrequencies can be used if a one-to-one correspondence can be constructed between the computational 
modes and the experimental modes $[45,5,2]$. This means that the experimental variability and the randomness in the SCM should not induce mode crossing phenomena or mode veering phenomena (see $[36,37,33]$ ) for the experimental modes and for the computed stochastic modes. This is true if the variability is sufficiently small and if the resonances are well-separated. If this is not the case, information on the mode shapes have to be used in order to construct a one-to-one correspondence between the experimental data and the corresponding computational quantities.

It should be noted that in industrial context, the quantification of the confidence in computational models must be established so that they can be used either in design purpose or in expertise purpose. For example, the system presented in the application can operate at design conditions, at offdesign conditions and at failure-mode conditions that apply in accident scenarios. An adapted way to take into account their generic characteristics and their capability to reproduce the behavior of the whole family of nominallyidentical structures is to consider no more deterministic but stochastic computational models, using experimental data. This is the main motivation for the development of the general approach proposed.

The objective of this paper consists in identifying the hyperparameters of a SCM using natural frequencies and mass-normalized mode shapes measured for a family of structures. The methodology proposed introduces a random transformation of the computational observations (computational eigenfrequencies and computational mode shapes) in order to match them to the experimental observation of each measured structure. This methodology automatically takes into account the mode crossings and the mode veerings which can appear between two experimental configurations or between two computational realizations of the SCM. In Section 2, the construction of the SCM is summarized. Section 3 is devoted to the identification of the hyperparameters of the SCM using a new methodology. Finally, in Section 4, an application devoted to an industrial pump of a thermal unit is presented.

\section{Construction of the stochastic computational model}

The construction of an adapted SCM can be carried out using different approaches (a state-of-the-art concerning stochastic modeling of uncertainties can be found, for instance, in $[39,40,48])$. The objective of this section is 
to construct a parameterized SCM based on the use of the generalized probabilistic approach of uncertainties proposed in [47, 48], for which both the model-parameter uncertainties and the model uncertainties are taken into account and are separately identified. First, the NCM is constructed using the Finite Element (FE) method. The probabilistic model of model-parameter uncertainties is constructed by replacing the uncertain model parameters by random variables and the probabilistic model of model uncertainties is constructed by replacing the mass and stiffness matrices by adapted random matrices. The prior probability distributions of the uncertain model parameters and of the random matrices depend on unknown parameters (called the hyperparameters) for which the identification is addressed in Section 3.

\subsection{Construction of the nominal computational model}

The NCM is constructed using the FE method and the boundary conditions of the structure are such that there are no rigid body modes. This computational model exhibits $n_{p}$ uncertain model parameters denoted $h_{1}, \ldots, h_{n_{p}}$. Let be $\mathbf{h}=\left(h_{1}, \ldots, h_{n_{p}}\right)$ belonging to an admissible set, $\mathcal{C}_{h}$. Let $[\mathbb{M}(\mathbf{h})]$ and $[\mathbb{K}(\mathbf{h})]$ be the mass and stiffness $(m \times m)$ real matrices of the NCM. For all $\mathbf{h}$ in $\mathcal{C}_{h}$, we are interested in calculating the first $n$ (with $n<m$ ) eigenvalues $0<\lambda_{1}(\mathbf{h}) \leq \ldots \leq \lambda_{n}(\mathbf{h})$ and the associated mode shapes, $\boldsymbol{\phi}_{1}(\mathbf{h}), \ldots, \boldsymbol{\phi}_{n}(\mathbf{h})$. These modal quantities are the solutions of the following generalized eigenvalue problem related to the NCM,

$$
[\mathbb{K}(\mathbf{h})] \boldsymbol{\phi}(\mathbf{h})=\lambda(\mathbf{h})[\mathbb{M}(\mathbf{h})] \boldsymbol{\phi}(\mathbf{h}) .
$$

Let $[\Phi(\mathbf{h})]$ be the $m \times n$ matrix whose columns are the first $n$ mode shapes. We then introduce the $n \times n$ mass and stiffness reduced matrices, $[M(\mathbf{h})]=$ $[\Phi(\mathbf{h})]^{T}[\mathbb{M}(\mathbf{h})][\Phi(\mathbf{h})]$ and $[K(\mathbf{h})]=[\Phi(\mathbf{h})]^{T}[\mathbb{K}(\mathbf{h})][\Phi(\mathbf{h})]$. For convenience, the mode shapes are normalized with respect to the mass matrix such that

$$
[M(\mathbf{h})]=\left[I_{n}\right],
$$

in which $\left[I_{n}\right]$ is the $n \times n$ identity matrix. The diagonal $n \times n$ real matrix $[K(\mathbf{h})]$ is then written as

$$
[K(\mathbf{h})]=\operatorname{diag}\left(\lambda_{1}(\mathbf{h}), \ldots, \lambda_{n}(\mathbf{h})\right)
$$




\subsection{Construction of the probabilistic model of uncertain model parameters}

The probabilistic model of uncertain model parameters is constructed by replacing vector $\mathbf{h}$ of the uncertain model parameters by the random vector $\mathbf{H}$ with values in $\mathbb{R}^{n_{p}}$, defined on a probability space $\left(\Theta^{\text {par }}, \mathcal{T}^{\text {par }}, \mathcal{P}^{\text {par }}\right)$ and for which the probability density function (pdf) $p_{\mathbf{H}}(\mathbf{h})$ with respect to $d \mathbf{h}$ depends on hyperparameters which can be mean values, coefficients of variation, Lagrange multipliers (in case of a Maximum Entropy construction $[42,25,26])$, and so on. The first $n$ random eigenvalues $0<\Lambda_{1}^{\text {par }} \leq \ldots \leq \Lambda_{n}^{\text {par }}$ associated with the random mode shapes $\phi_{1}^{\text {par }}, \ldots, \phi_{n}^{\text {par }}$ are the solutions of the following random generalized eigenvalue problem,

$$
[\mathbb{K}(\mathbf{H})] \phi^{\mathrm{par}}=\Lambda^{\mathrm{par}}[\mathbb{M}(\mathbf{H})] \boldsymbol{\phi}^{\mathrm{par}} .
$$

Let $\left[\boldsymbol{\Phi}^{\text {par }}\right]$ be the $m \times n$ random matrix whose columns are the first $n$ random mode shapes. We then introduce the $n \times n$ random mass and stiffness reduced matrices $\left[\mathbf{M}^{\mathrm{par}}\right]=\left[\boldsymbol{\Phi}^{\mathrm{par}}\right]^{T}[\mathbb{M}(\mathbf{H})]\left[\boldsymbol{\Phi}^{\mathrm{par}}\right]$ and $\left[\mathbf{K}^{\mathrm{par}}\right]=\left[\boldsymbol{\Phi}^{\mathrm{par}}\right]^{T}[\mathbb{K}(\mathbf{H})]\left[\boldsymbol{\Phi}^{\mathrm{par}}\right]$. The random mode shapes are normalized (almost surely) with respect to the random mass matrix such that

$$
\left[\mathbf{M}^{\mathrm{par}}\right]=\left[I_{n}\right]
$$

and thus, the random diagonal $n \times n$ real matrix $\left[\mathbf{K}^{\text {par }}\right]$ is written as

$$
\left[\mathbf{K}^{\text {par }}\right]=\operatorname{diag}\left(\Lambda_{1}^{\text {par }}, \ldots, \Lambda_{\mathrm{n}}^{\text {par }}\right) .
$$

By construction, the random matrices $\left[\mathbf{M}^{\text {par }}\right]$ and $\left[\mathbf{K}^{\text {par }}\right]$ are positive definite (almost surely) and therefore, their Cholesky decompositions yield,

$$
\left[\mathbf{M}^{\mathrm{par}}\right]=\left[\mathbf{L}_{M}\right]^{T}\left[\mathbf{L}_{M}\right], \quad\left[\mathbf{K}^{\mathrm{par}}\right]=\left[\mathbf{L}_{K}\right]^{T}\left[\mathbf{L}_{K}\right] .
$$

From Eqs. (5) to (7), it can be deduced that $\left[\mathbf{L}_{M}\right]=\left[I_{m}\right]$ and $\left[\mathbf{L}_{K}\right]=$ $\operatorname{diag}\left(\Omega_{1}^{\mathrm{par}}, \ldots, \Omega_{n}^{\mathrm{par}}\right)$ where $\Omega_{j}^{\mathrm{par}}=\sqrt{\Lambda_{j}^{\mathrm{par}}}$ is the $j^{\text {th }}$ random eigenfrequency (in $\mathrm{rad} / \mathrm{s}$ ).

Let $\boldsymbol{\alpha}_{\mathrm{par}}$ be the vector whose components are the hyperparameters of the pdf $p_{\mathbf{H}}(\mathbf{h})$ which is then rewritten as $p_{\mathbf{H}}\left(\mathbf{h} ; \boldsymbol{\alpha}_{\mathrm{par}}\right)$. The vector-valued hyperparameter $\boldsymbol{\alpha}_{\text {par }}$ must be identified using experimental modal data. 


\subsection{Construction of the generalized probabilistic model of uncertainties}

Let $\left(\Theta^{\text {mod }}, \mathcal{T}^{\text {mod }}, \mathcal{P}^{\text {mod }}\right)$ be another probability space. To take into account model uncertainties (induced by modeling errors), the dependent random matrices $\left[\mathbf{M}^{\mathrm{par}}\right]$ and $\left[\mathbf{K}^{\mathrm{par}}\right]$ are replaced by the dependent random matrices $\left[\mathbf{M}^{\text {tot }}\right],\left[\mathbf{K}^{\text {tot }}\right]$, defined on a probability space $\left(\Theta=\Theta^{\text {par }} \times \Theta^{\text {mod }}, \mathcal{T}=\right.$ $\left.\mathcal{T}^{\text {par }} \otimes \mathcal{T}^{\text {mod }}, \mathcal{P}=\mathcal{P}^{\text {par }} \otimes \mathcal{P}^{\text {mod }}\right)$, such that for all $\theta=\left(\theta^{\text {par }}, \theta^{\text {mod }}\right)$ in $\Theta=$ $\Theta^{\text {par }} \times \Theta^{\text {mod }}$

$$
\begin{array}{r}
{\left[\mathbf{M}^{\text {tot }}(\theta)\right]=\left[\mathbf{L}_{M}\left(\theta^{\text {par }}\right)\right]^{T}\left[\mathbf{G}_{M}\left(\theta^{\text {mod }}\right)\right]\left[\mathbf{L}_{M}\left(\theta^{\text {par }}\right)\right],} \\
{\left[\mathbf{K}^{\text {tot }}(\theta)\right]=\left[\mathbf{L}_{K}\left(\theta^{\text {par }}\right)\right]^{T}\left[\mathbf{G}_{K}\left(\theta^{\text {mod }}\right)\right]\left[\mathbf{L}_{K}\left(\theta^{\text {par }}\right)\right],}
\end{array}
$$

in which the probability distributions of the random matrices $\left[\mathbf{G}_{M}\right]$ and $\left[\mathbf{G}_{K}\right]$, defined on $\left(\Theta^{\text {mod }}, \mathcal{T}^{\text {mod }}, \mathcal{P}^{\text {mod }}\right)$, are explicitly given in [44] in the context of the nonparametric probabilistic approach of uncertainties. The probability distributions of $\left[\mathbf{G}_{M}\right]$ and $\left[\mathbf{G}_{K}\right]$ depend on the dispersion parameters $\delta_{M}$ and $\delta_{K}$ respectively. Let $\boldsymbol{\alpha}_{\text {mod }}$ be the vector of the dispersion parameters such that $\boldsymbol{\alpha}_{\text {mod }}=\left(\delta_{M}, \delta_{K}\right)$.

The random matrices $\left[\mathbf{M}^{\text {tot }}\right]$ and $\left[\mathbf{K}^{\text {tot }}\right]$ are not diagonal. In order to calculate the random eigenfrequencies and the random mode shapes of the SCM with both the model-parameter uncertainties and the model uncertainties, the following small-dimension random generalized eigenvalue problem is introduced. Let $0<\Lambda_{1} \leq \ldots \leq \Lambda_{n}$ be the first $n$ random eigenvalues associated with the random eigenvectors $\boldsymbol{\phi}_{1}^{\text {tot }}, \ldots, \boldsymbol{\phi}_{n}^{\text {tot }}$ which are the solutions of the following random reduced generalized eigenvalue problem

$$
\left[\mathbf{K}^{\mathrm{tot}}\right] \boldsymbol{\phi}^{\mathrm{tot}}=\Lambda\left[\mathbf{M}^{\mathrm{tot}}\right] \boldsymbol{\phi}^{\mathrm{tot}}
$$

Let $\left[\boldsymbol{\Phi}^{\text {tot }}\right]=\left[\boldsymbol{\phi}_{1}^{\text {tot }}, \ldots, \phi_{n}^{\text {tot }}\right]$. These random modes are normalized with respect to the random mass matrix $\left[\mathbf{M}^{\text {tot }}\right]$,

$$
[\mathbf{M}]=\left[\boldsymbol{\Phi}^{\mathrm{tot}}\right]^{T}\left[\mathbf{M}^{\mathrm{tot}}\right]\left[\boldsymbol{\Phi}^{\mathrm{tot}}\right]=\left[I_{n}\right]
$$

and we have

$$
[\mathbf{K}]=\left[\boldsymbol{\Phi}^{\mathrm{tot}}\right]^{T}\left[\mathbf{K}^{\mathrm{tot}}\right]\left[\boldsymbol{\Phi}^{\mathrm{tot}}\right]=\operatorname{diag}\left(\Lambda_{1}, \ldots, \Lambda_{n}\right) .
$$

Then the first $n$ random eigenvalues of the SCM, with both the modelparameter uncertainties and the model uncertainties, are $0<\Lambda_{1} \leq \ldots \leq \Lambda_{n}$ and the associated random vectors are $\phi_{1}, \ldots, \phi_{n}$ such that the $m \times n$ random 
matrix $[\boldsymbol{\Phi}]=\left[\boldsymbol{\phi}_{1}, \ldots, \boldsymbol{\phi}_{n}\right]$ is written as

$$
[\Phi]=\left[\boldsymbol{\Phi}^{\text {par }}\right]\left[\boldsymbol{\Phi}^{\text {tot }}\right]
$$

Finally the SCM is parameterized by the vector-valued hyperparameter $\boldsymbol{\alpha}=$ $\left(\boldsymbol{\alpha}_{\text {par }}, \boldsymbol{\alpha}_{\text {mod }}\right)$ which has to be identified using experimental modal data. The admissible space for vector $\boldsymbol{\alpha}$ is denoted by $\mathcal{C}$.

\section{Identification of the SCM using experimental modal data}

The objective of this section is to identify the parameter $\boldsymbol{\alpha}$ of the SCM using experimental modal data (eigenfrequencies and mass-normalized mode shapes) and realizations of the modal data calculated using the SCM.

\subsection{Experimental modal data as observations}

It is assumed that $n_{\text {exp }}$ experimental configurations of the structure (simply called "configurations") have been tested. For each configuration $j, n_{j}$ modes have been experimentally identified using an experimental modal analysis method (see [18, 32]). For two given configurations, the number of modes, the number and locations of the sensors can be different. For each configuration $j, n_{j}$ experimental eigenfrequencies $\omega_{1}^{\text {exp }, \mathrm{j}}, \ldots, \omega_{n_{j}}^{\text {exp } \mathrm{j}}$ associated with $n_{j}$ mass-normalized experimental mode shapes $\widehat{\varphi}_{1}^{\exp , \mathrm{j}}, \ldots, \widehat{\varphi}_{n_{j}}^{\exp , \mathrm{j}}$ have been iden-

tified for $m_{j}$ degrees of freedom (DOFs). Let $\left[\widehat{\Phi}^{\exp , \mathrm{j}}\right]=\left[\widehat{\varphi}_{1}^{\exp , \mathrm{j}} \ldots \widehat{\varphi}_{n_{j}, \mathrm{j}}^{\exp }\right]$ be the $m_{j} \times n_{j}$ matrix of the $n_{j}$ experimental mode shapes of the configuration $j$. It is assumed that $n_{j}<n<m_{j}<m$ for all $j$ in $\left\{1, \ldots, n_{\text {exp }}\right\}$. The experimental reduced mass matrix and the experimental reduced stiffness matrix are then written as

$$
\left[\widehat{M}^{\text {exp,j}}\right]=\left[I_{n_{j}}\right] \quad, \quad\left[\widehat{K}^{\exp , \mathrm{j}}\right]=\operatorname{diag}\left(\lambda_{1}^{\exp , \mathrm{j}}, \ldots, \lambda_{n_{j}}^{\exp , \mathrm{j}}\right)
$$

in which $\lambda_{i}^{\exp , \mathrm{j}}=\left(\omega_{i}^{\exp , \mathrm{j}}\right)^{2}$.

\subsection{Transformation of the modal data}

For all $j$ in $\left\{1, \ldots, n_{\text {exp }}\right\}$, let $\left[P^{\mathrm{j}}\right]$ be the $m_{j} \times m$ matrix that performs the projection from the $m$ DOFs of the SCM to the $m_{j}$ DOFs of the experimental configuration $j$. Then the projected random modal basis $\left[\widetilde{\boldsymbol{\Phi}}^{\mathrm{j}}\right]$ of the SCM is defined by

$$
\left[\widetilde{\boldsymbol{\Phi}}^{\mathrm{j}}\right]=\left[P^{\mathrm{j}}\right][\boldsymbol{\Phi}]
$$


The experimental modes $\left[\widehat{\boldsymbol{\Phi}}^{\text {exp,j}}\right]$ cannot directly be compared to the computational modes $\left[\widetilde{\boldsymbol{\Phi}}^{\mathrm{j}}\right]$ because, in general, there is not a one-to-one correspondence between the experimental modes and the computational modes. Indeed, some modes may be missing in the experimental modal basis or in the computational modal basis. Furthermore, due to the experimental variability (variability of the configurations) and the computational randomness (uncertainties), some mode crossing and/or mode veering [36, 37, 33] phenomena may occur. The classical mode tracking methods [28, 11] based on the MAC criterion [18] are efficient to detect mode missings and mode crossings but are not able to detect mode veerings (see [1], [33]). In Ref. [1], in the context of the interpolation of reduced-order models (ROM), the authors have introduced a transformation for a given ROM, $\{[\widetilde{\Phi}],[K],[M]\}$, in which $[\widetilde{\Phi}]$ is a reduced-order basis, $[K]$ is a reduced stiffness matrix and $[M]$ is a reduced mass matrix, in order to match it with a reference $\mathrm{ROM},\left\{\left[\widehat{\Phi}^{\mathrm{ref}}\right],\left[K^{\mathrm{ref}}\right],\left[M^{\mathrm{ref}}\right]\right\}$, of the same dimension. The transformed ROM is constructed using a congruence transformation and is then written as $\left\{[\widetilde{\Phi}][Q],[Q]^{T}[K][Q],[Q]^{T}[M][Q]\right\}$, in which $[Q]$ is a square orthogonal matrix that minimizes the distance $\left\|[\widetilde{\Phi}][Q]-\left[\widehat{\Phi}^{\text {ref }}\right]\right\|_{F}$, where $\|.\|_{F}$ is the Frobenius norm. Such a transformation is also used in the context of modal expansion methods (see $[43,29]$ for instance). In the present paper, a similar method is introduced in order to construct a one-to-one correspondence between the random ROM calculated using the SCM and the experimental ROM.

For each experimental configuration $j$, the above reference ROM is chosen as the experimental ROM, $\left\{\left[\widehat{\Phi}^{\exp , \mathrm{j}}\right],\left[\widehat{K}^{\text {exp,j }}\right],\left[\widehat{M}^{\text {exp,j }}\right]\right\}$. The ROM to be transformed is the projected computational ROM, $\left\{\left[\widetilde{\mathbf{\Phi}}^{\mathrm{j}}\right],[\mathbf{K}],[\mathbf{M}]\right\}$ which is not of the same dimension as the experimental ROM. Therefore, the projected computational ROM, $\left\{\left[\widetilde{\mathbf{\Phi}}^{\mathrm{j}}\right],[\mathbf{K}],[\mathbf{M}]\right\}$, is transformed into the following ROM, $\left\{\left[\widehat{\boldsymbol{\Phi}}^{\mathrm{j}}\right],\left[\widehat{\mathbf{K}}^{\mathrm{j}}\right],\left[\widehat{\mathbf{M}}^{\mathrm{j}}\right]\right\}$, such that

$$
\begin{gathered}
{\left[\widehat{\boldsymbol{\Phi}}^{\mathrm{j}}\right]=\left[\widetilde{\boldsymbol{\Phi}}^{\mathrm{j}}\right]\left[\mathbf{Q}^{\mathrm{opt}, \mathrm{j}}\right],} \\
{\left[\widehat{\mathbf{K}}^{\mathrm{j}}\right]=\left[\mathbf{Q}^{\mathrm{opt}, \mathrm{j}}\right]^{T}[\mathbf{K}]\left[\mathbf{Q}^{\mathrm{opt}, \mathrm{j}}\right],} \\
{\left[\widehat{\mathbf{M}}^{\mathrm{j}}\right]=\left[\mathbf{Q}^{\mathrm{opt}, \mathrm{j}}\right]^{T}[\mathbf{M}]\left[\mathbf{Q}^{\mathrm{opt}, \mathrm{j}}\right],}
\end{gathered}
$$

in which $\left[\mathbf{Q}^{\mathrm{opt}, \mathrm{j}}\right]$ is a random $n \times n_{j}$ real matrix for which each realization $[Q]=$ 
$\left[\mathbf{Q}^{\mathrm{opt}, \mathrm{j}}(\theta)\right]$, for $\theta$ in $\Theta$, must belong to the Stiefel manifold [16], $\mathcal{O S} t\left(n, n_{j}\right)$, defined by

$$
\mathcal{O S t}\left(n, n_{j}\right)=\left\{[Q] \in \mathbb{R}^{n \times n_{j}},[Q]^{T}[Q]=\left[I_{n_{j}}\right]\right\} .
$$

We then have the following properties for the random $n_{j} \times n_{j}$ real matrices, $\left[\widehat{\mathbf{K}}^{\mathrm{j}}\right]$ and $\left[\widehat{\mathbf{M}}^{\mathrm{j}}\right]$,

$$
\begin{array}{r}
{\left[\widehat{\mathbf{K}}^{\mathrm{j}}\right] \text { is with values in } \mathbb{M}_{n_{j}}^{+}(\mathbb{R}),} \\
{\left[\widehat{\mathbf{M}}^{\mathrm{j}}\right]=\left[I_{n_{j}}\right],}
\end{array}
$$

in which $\mathbb{M}_{n_{j}}^{+}(\mathbb{R})$ is the set of all the positive-definite $n_{j} \times n_{j}$ real matrices. For all $\theta$ in $\Theta$, orthogonal matrix $\left[\mathbf{Q}^{\mathrm{opt}, \mathrm{j}}(\theta)\right]$ is calculated by minimizing the distance between the computational modal basis $\left[\widehat{\boldsymbol{\Phi}}^{\mathrm{j}}(\theta)\right]$ and the experimental modal basis $\left[\widehat{\Phi}^{\mathrm{exp}, \mathrm{j}}\right]$,

$$
\left[\mathbf{Q}^{\mathrm{opt}, \mathrm{j}}(\theta)\right]=\underset{[Q] \in \mathcal{O} \mathcal{S} t\left(n, n_{j}\right)}{\arg } \min \left\|\left[\widetilde{\boldsymbol{\Phi}}^{\mathbf{j}}(\theta)\right][Q]-\left[\widehat{\Phi}^{\mathrm{exp}, \mathbf{j}}\right]\right\|_{F}
$$

The minimization problem (14) is referred as a Procruste problem $[7,8]$. Contrary to a classical Procruste problem (for which $[Q]$ is searched in the square orthogonal matrices ensemble [24]), this Procruste problem does not have an explicit solution. Nevertheless, a solution can be calculated iteratively (see [7]) using the Algorithm 1. The number of iterations $n_{\text {iter1 }}$ is determined through a convergence analysis of the error $\left\|\left[\widetilde{\boldsymbol{\Phi}}^{\mathrm{j}}(\theta)\right]\left[\mathbf{Q}^{\mathrm{opt}, \mathrm{j}}(\theta)\right]-\left[\widehat{\Phi}^{\mathrm{exp}, \mathrm{j}}\right]\right\|_{F}$. In general, this error does not converge to zero. This convergence analysis can be performed for all the realizations $\left[\widetilde{\boldsymbol{\Phi}}^{\mathrm{j}}(\theta)\right]$ or can be performed only one time using the mode shapes of the NCM. In this case, the transformed nominal $\operatorname{ROM}\{[\widehat{\Phi}(\mathbf{h})],[\widehat{K}(\mathbf{h})],[\widehat{M}(\mathbf{h})]\}$ such that

$$
\begin{gathered}
{\left[\widehat{\Phi}^{\mathrm{j}}(\mathbf{h})\right]=\left[\widetilde{\Phi}^{\mathrm{j}}(\mathbf{h})\right]\left[Q^{\mathrm{opt}, \mathrm{j}}(\mathbf{h})\right],} \\
{\left[\widehat{K}^{\mathrm{j}}(\mathbf{h})\right]=\left[Q^{\mathrm{opt}, \mathrm{j}}(\mathbf{h})\right]^{T}[K(\mathbf{h})]\left[Q^{\mathrm{opt}, \mathrm{j}}(\mathbf{h})\right],} \\
{\left[\widehat{M}^{\mathrm{j}}(\mathbf{h})\right]=\left[Q^{\mathrm{opt}, \mathrm{j}}(\mathbf{h})\right]^{T}[M(\mathbf{h})]\left[Q^{\mathrm{opt}, \mathrm{j}}(\mathbf{h})\right],}
\end{gathered}
$$

in which $\left[\widetilde{\Phi}^{\mathrm{j}}(\mathbf{h})\right]=\left[P^{\mathrm{j}}\right][\Phi(\mathbf{h})]$ and $\left[Q^{\mathrm{opt}, \mathrm{j}}(\mathbf{h})\right]$ is calculated by minimizing the distance between the nominal modal basis $\left[\widehat{\Phi}^{\mathrm{j}}(\mathbf{h})\right]$ and the experimental modal basis $\left[\widehat{\Phi}^{\exp , \mathrm{j}}\right]$. 


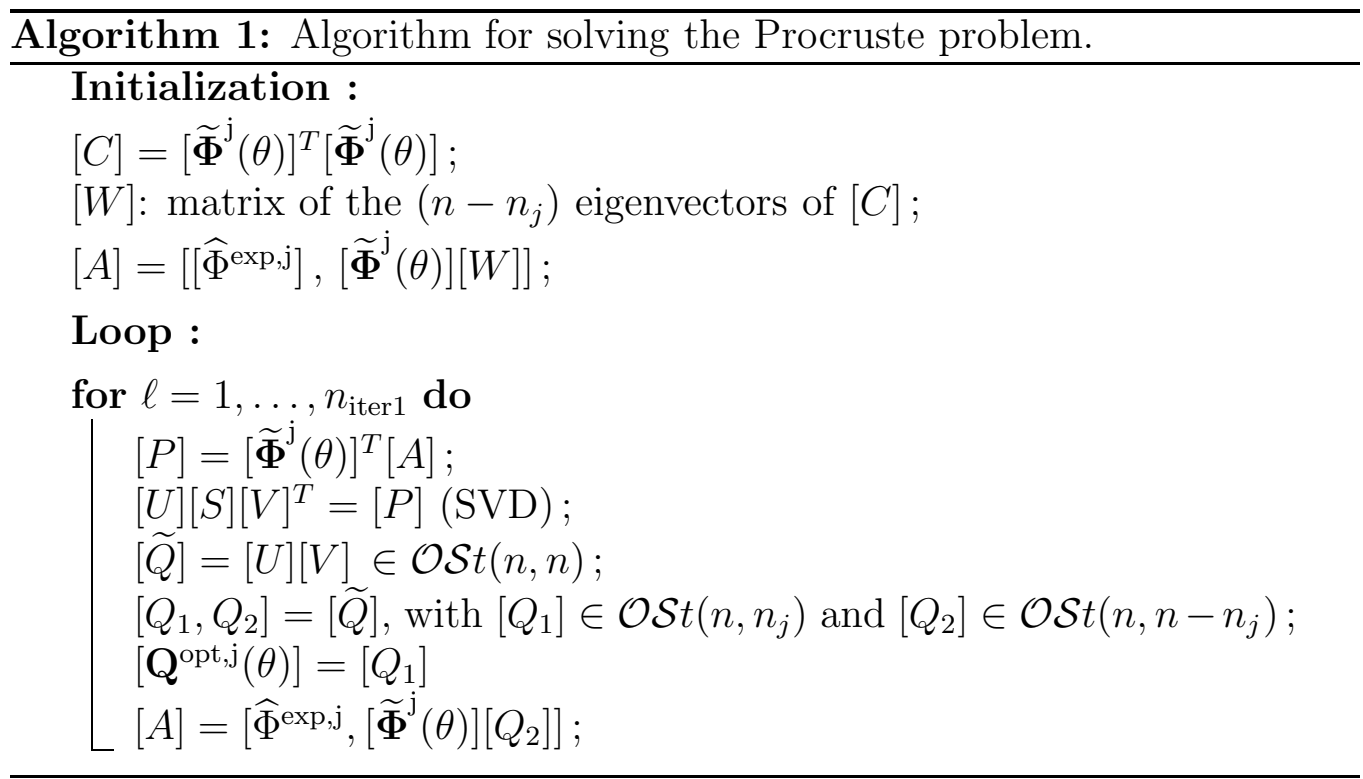

If the experimental mode shapes $\widehat{\varphi}_{1}^{\exp , \mathrm{j}}, \ldots, \widehat{\varphi}_{n_{\text {exp }}, \mathrm{j}}^{\exp }$ are not mass-normalized (because the modal masses have not been identified with a good precision) then the transformation defined by Eqs. (15) to (20) is not enough to make the random computational mode shapes close enough to the experimental mode shapes. It is then necessary to include an external scaling loop to Algorithm 1 so that the columns of matrices $\left[\widehat{\Phi}^{\exp , \mathrm{j}}\right]$ and $\left[\widehat{\mathbf{\Phi}}^{\mathrm{j}}(\theta)\right]=\left[\widetilde{\boldsymbol{\Phi}}^{\mathrm{j}}(\theta)\right]\left[\mathbf{Q}^{\mathrm{opt}, \mathrm{j}}(\theta)\right]$ have the same norm. This external loop is summarized in Algorithm 2.

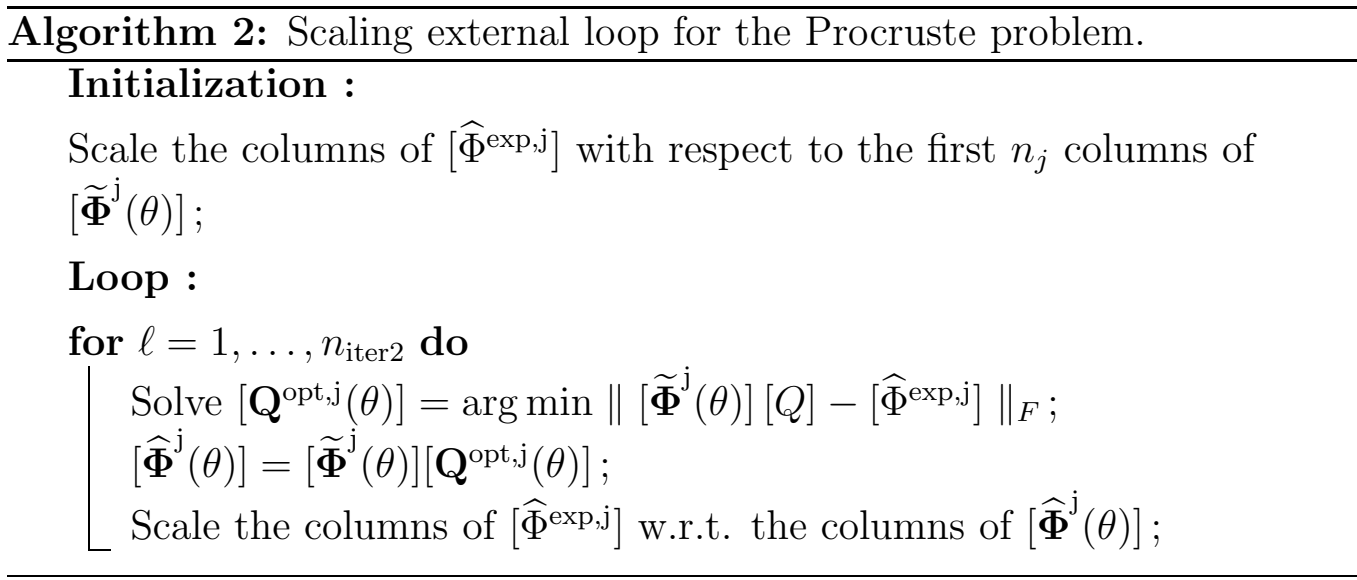

It should be noted that, in [2], the authors have proposed an alterna- 
tive solution to take into account mode crossings and mode veerings in the identification of the hyperparameters of a SCM. Their solution consists in projecting the experimental mode shapes in the modal basis of the NCM and in letting the eigenfrequencies unchanged. A similar procedure has recently been proposed in [10].

\subsection{Computational modal quantities as observations}

For $j=1, \ldots, n_{\text {exp }}$, the experimental $\operatorname{ROM}\left\{\left[\widehat{\Phi}^{\exp , \mathrm{j}}\right],\left[\widehat{K}^{\exp , \mathrm{j}}\right],\left[\widehat{M}^{\exp , \mathrm{j}}\right]\right\}$ has to be compared to the projected computational ROM, $\left\{\left[\widehat{\boldsymbol{\Phi}}^{\mathrm{j}}\right],\left[\widehat{\mathbf{K}}^{\mathrm{j}}\right],\left[\widehat{\mathbf{M}}^{\mathrm{j}}\right]\right\}$, calculated using the SCM. Since $\left[\widehat{\mathbf{M}}^{\mathrm{j}}\right]=\left[\widehat{M}^{\mathrm{exp}, \mathrm{j}}\right]=\left[I_{n_{j}}\right]$, only the mode shapes and the stiffness matrices have to be compared. Before proceeding to the identification, it is convenient to represent the experimental modal data and the corresponding computational modal quantities in an Euclidean space. Such a representation can be achieved through logarithm maps [1]. Concerning the modal basis, we define $\left[\widehat{\boldsymbol{\Phi}}^{\text {obs,j }}\right]$ such that

$$
\left[\widehat{\boldsymbol{\Phi}}^{\mathrm{obs}, \mathrm{j}}\right]=\left[\widehat{\boldsymbol{\Phi}}^{\mathrm{j}}\right]-\left[\widehat{\Phi}^{\mathrm{exp}, \mathrm{j}}\right]
$$

and the corresponding experimental observation $\left[\widehat{\Phi}^{\text {exp,obs,j] }}\right]$ is defined by

$$
\left[\widehat{\Phi}^{\exp , \mathrm{obs}, \mathrm{j}}\right]=[0] .
$$

Concerning the stiffness matrix, we define $\left[\widehat{\mathbf{K}}_{\operatorname{logm}}^{\text {obs,j }}\right]$ such that

$$
\left[\widehat{\mathbf{K}}_{\operatorname{logm}}^{\mathrm{obs}, \mathrm{j}}\right]=\operatorname{logm}\left(\left[\widehat{\mathrm{K}}^{\mathrm{exp}, \mathrm{j}}\right]^{-\frac{1}{2}}\left[\widehat{\mathbf{K}}^{\mathrm{j}}\right]\left[\widehat{\mathrm{K}}^{\mathrm{exp}, \mathrm{j}}\right]^{-\frac{1}{2}}\right),
$$

in which logm is the logarithm for matrices. The corresponding experimental observation $\left[\widehat{K}_{\operatorname{logm}}^{\text {exp }, \text { obs,j }}\right]$ is such that

$$
\left[\widehat{K}_{\log m}^{\exp , \mathrm{obs}, \mathrm{j}}\right]=[0],
$$

Let $n_{o b s, j}=\left(m_{j} \times n_{j}+n_{j} \times\left(n_{j}+1\right) / 2\right)$. We then introduce the random observation vector, $\mathbf{W}^{j}$, with values in $\mathbb{R}^{n_{o b s, j}}$ containing the $m_{j} \times n_{j}$ entries of random matrix $\left[\widehat{\boldsymbol{\Phi}}^{\mathrm{obs}, \mathrm{j}}\right]$ and the $n_{j} \times\left(n_{j}+1\right) / 2$ entries of the upper triangular part of random matrix $\left[\widehat{\mathbf{K}}_{\operatorname{logm}}^{\mathrm{obs}, \mathrm{j}}\right]$. The corresponding experimental observation vector is zero. For the observations, this representation has the advantage to belong to Euclidean space $\mathbb{R}^{n_{o b s, j}}$ and therefore, the probability distribution 
of the vector-valued random observation will be easier to estimate since its support is not a complex manifold.

\subsection{Identification of hyperparameter $\boldsymbol{\alpha}$}

Hyperparameter $\boldsymbol{\alpha}$ of the SCM is identified using the maximum likelihood method and experimental modal data. Then the optimal values $\boldsymbol{\alpha}^{\text {opt }}$ is solution of the following optimization problem

$$
\boldsymbol{\alpha}^{\text {opt }}=\arg \max _{\boldsymbol{\alpha} \in \mathcal{C}} \sum_{j=1}^{n_{\exp }} \log \left(p_{\mathbf{w}^{j}}(\mathbf{0} ; \boldsymbol{\alpha})\right),
$$

where $p_{\mathbf{w}^{j}}(\mathbf{w} ; \boldsymbol{\alpha})$ is the probability density function (pdf) of random vector $\mathbf{W}^{j}$. This pdf is estimated using the Monte Carlo simulation method [38] and a multi-dimensional kernel density estimator (see [52]). As proposed in [47], a statistical reduction can be performed for random vector $\mathbf{W}^{j}$ in order to reduce the statistical dimension of the random observation and then to facilitate the estimation of its pdf. Furthermore, a good predictor of $\boldsymbol{\alpha}^{\text {opt }}$ can be obtained by assuming a Gaussian pdf for random vector $\mathbf{W}^{j}$. Since random vector $\mathbf{W}^{j}$ is with values in $\mathbb{R}^{n_{o b s, j}}$, such an assumption is admissible. For the predictor, the optimization problem defined by Eq. (28) is then replaced by the following one

$$
\begin{aligned}
\mathcal{L}(\boldsymbol{\alpha})=\sum_{j=1}^{n_{\text {exp }}}\left\{-n_{\mathrm{obs}, \mathrm{j}} \log (2 \pi)-\frac{1}{2} \log (\right. & \left.\operatorname{det}\left(\left[C_{W^{j}}(\boldsymbol{\alpha})\right]\right)\right) \\
& \left.-\frac{1}{2} \underline{\mathbf{w}}^{j}(\boldsymbol{\alpha})^{T}\left[C_{W^{j}}(\boldsymbol{\alpha})\right]^{-1} \underline{\mathbf{w}}^{j}(\boldsymbol{\alpha})\right\},
\end{aligned}
$$

in which the mean value $\underline{\mathbf{w}}^{j}$ and the covariance matrix $\left[C_{W^{j}}(\boldsymbol{\alpha})\right]$ of random vector $\mathbf{W}^{j}$ are estimated using the Monte Carlo simulation method. In high dimension, the determinant of the covariance matrix can be calculated using the eigenvalues $\left\{\sigma_{k}^{j}(\boldsymbol{\alpha}), k=1, \ldots, n_{\mathrm{obs}, \mathrm{j}}\right\}$ of the covariance matrix $\left[C_{W^{j}}(\boldsymbol{\alpha})\right]$. In this case, the optimization problem defined by Eq. (29) can be rewritten 


$$
\begin{aligned}
\mathcal{L}(\boldsymbol{\alpha})=\sum_{j=1}^{n_{\text {exp }}}\left\{-n_{\mathrm{obs}, \mathrm{j}} \log (2 \pi)-\frac{1}{2} \sum_{k=1}^{n_{\text {obs }}}\right. & \log \left(\sigma_{k}^{j}(\boldsymbol{\alpha})\right) \\
& \left.-\frac{1}{2} \underline{\mathbf{w}}^{j}(\boldsymbol{\alpha})^{T}\left[C_{W^{j}}(\boldsymbol{\alpha})\right]^{-1} \underline{\mathbf{w}}^{j}(\boldsymbol{\alpha})\right\} .
\end{aligned}
$$

In general, the optimization problem defined by Eq. (28) (or by Eq. (29) is non-convex and has to be solved using evolutionary algorithms and random search algorithm (see $[51,17,50]$ and, for example, [30] for an application to model updating in structural dynamics).

\section{Application}

\subsection{Industrial mechanical system and experimental modal data}

We are interested in the dynamical behavior of a one-stage booster pump used by Electricité de France (EDF) company in its thermal units (see Fig. 1). This pump is made up of a diffuser and a volute, with axial suction and vertical delivery, and is mounted on a metallic frame. It has been designed forty years ago by Sulzer Pumps. An experimental modal analysis has been

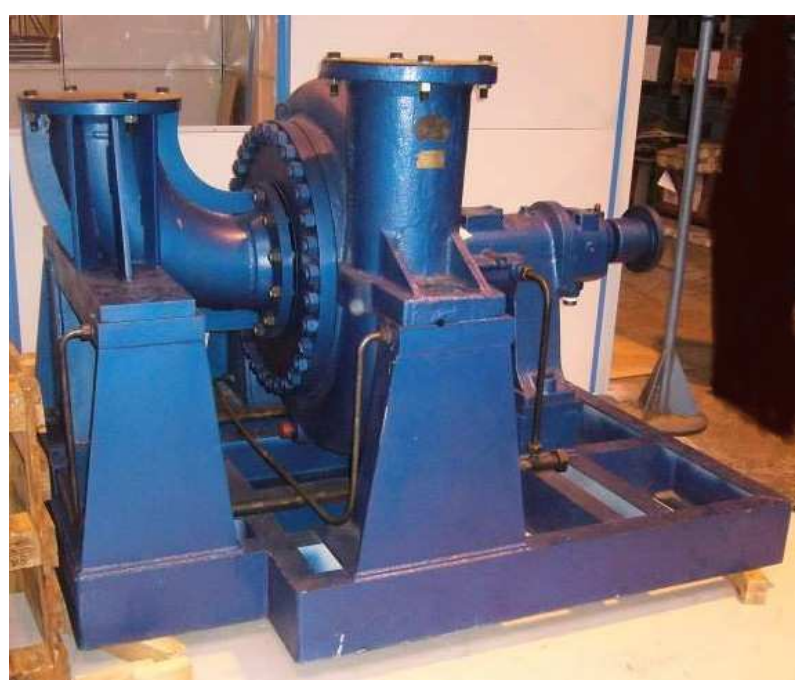

Figure 1: Industrial mechanical system. 
carried out on two specimens of this pump located at two different thermal units. Therefore, there are $n_{\text {exp }}=2$ experimental configurations (denoted as Pump 1 and Pump 2) which are measured. There are slight differences between Pump 1 and Pump 2 concerning the joints between the pumps and their metallic frame. The pumps have been disconnected to the adjacent piping before being equipped. The experimental meshes for the two pumps are not the same and are represented in Fig. 2. Pump 1 and Pump 2 have respectively been equipped with 55 and 113 triaxial accelerometers. Therefore $m_{1}=165$ and $m_{2}=339$. An experimental modal analysis has been
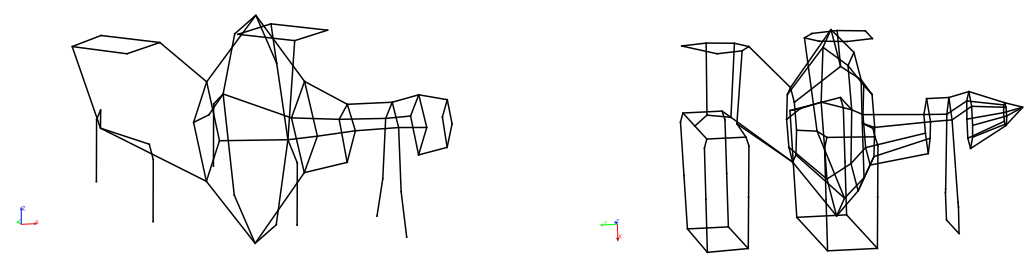

Figure 2: Experimental meshes: Pump 1 (left) and pump 2 (right).

carried for each pump. For Pump 1, $n_{1}=6$ modes have been identified with eigenfrequencies $76.8 \mathrm{~Hz}, 134.4 \mathrm{~Hz}, 162.0 \mathrm{~Hz}, 211.9 \mathrm{~Hz}, 233.4 \mathrm{~Hz}$ and 251.2 Hz. For Pump 2, $n_{2}=11$ modes have been identified with eigenfrequencies $69.9 \mathrm{~Hz}, 84.6 \mathrm{~Hz}, 151.8 \mathrm{~Hz}, 160.6 \mathrm{~Hz}, 166.3 \mathrm{~Hz}, 167.8 \mathrm{~Hz}, 202.5 \mathrm{~Hz}$, $224.6 \mathrm{~Hz}, 244.3 \mathrm{~Hz}, 252.1 \mathrm{~Hz}$ and $288.3 \mathrm{~Hz}$. The first six mode shapes for Pump 1 and Pump 2 are plotted in Figs. 3 and 4 respectively. In these
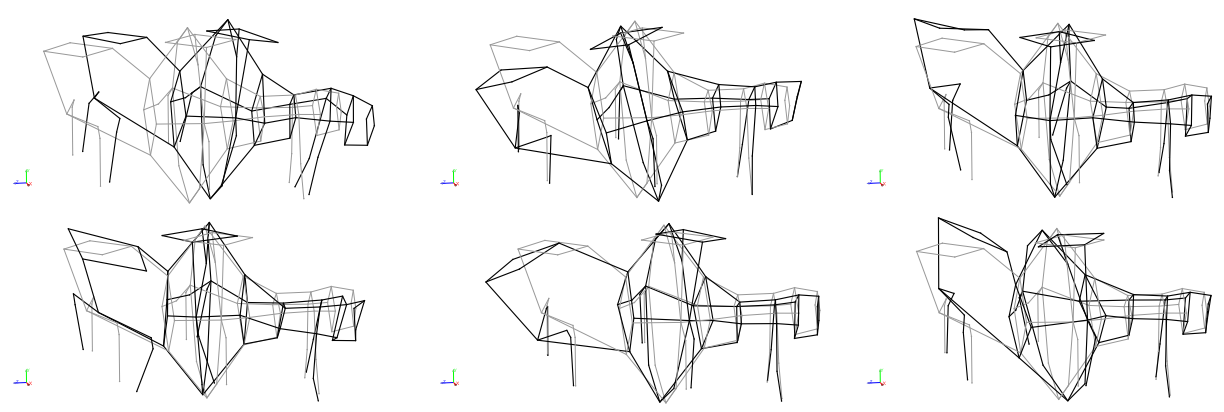

Figure 3: Pump 1: First six experimental mode shapes (Thick black line).

figures, it can be seen that, except for the first mode, it is not easy to make 

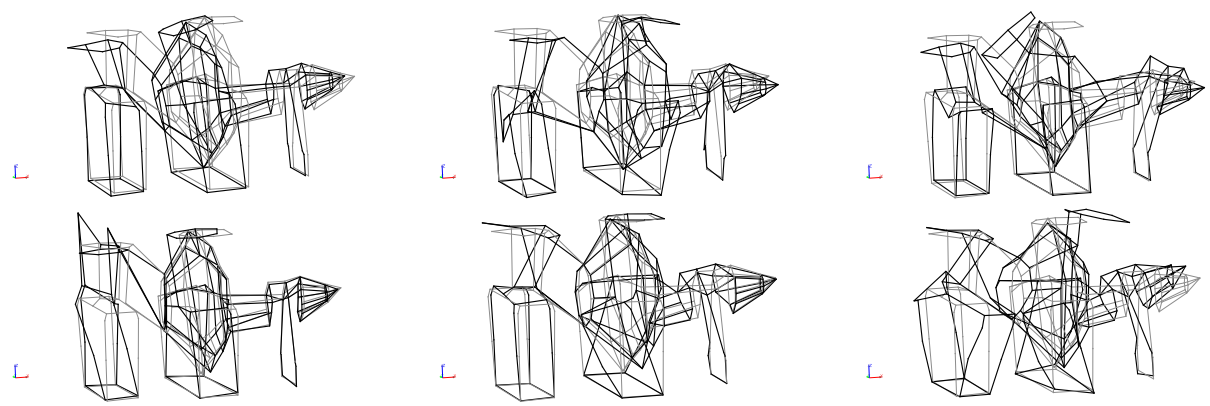

Figure 4: Pump 2: First six experimental mode shapes (Thick black line).

a correspondence between the modes of Pump 1 and the modes of Pump 2. This is due the variability of the configurations (as explained in the introduction). For both Pump 1 and Pump 2, the experimental mode shapes are not normalized with respect to the mass.

\subsection{Construction of the stochastic computational model}

\subsubsection{Construction of the nominal computational model}

The finite element mesh of the NCM is plotted in Fig. 5. The nominal

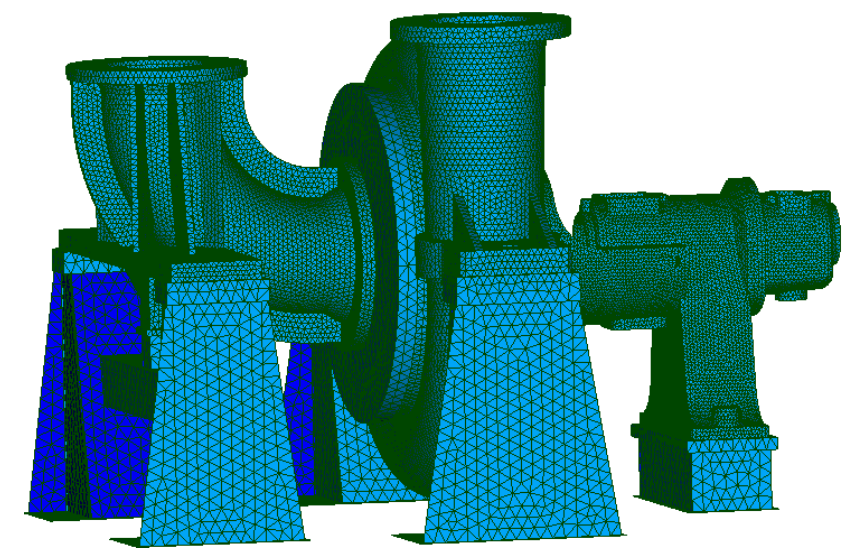

Figure 5: Finite element mesh of the NCM.

finite element model is made up of 3D solid elements, Kirchhoff plate elements and spring elements. The assembled model has 488, 220 DOFs. The uncertain model parameters of the NCM are the Young modulus $y_{s}$ of the 
steel, the Young's modulus $y_{c}$ of the cast iron, the thicknesses $t_{1}, t_{2}$ and $t_{3}$ of the plates 1, 2 and 3 of the metallic frame (see Fig. 6) and the stiffnesses $k_{1}, k_{2}, k_{3}$ and $k_{4}$ of the discrete springs normal to the metallic frame (see Fig. 6). The NCM for Pump 1 and the NCM for Pump 2 are the same but

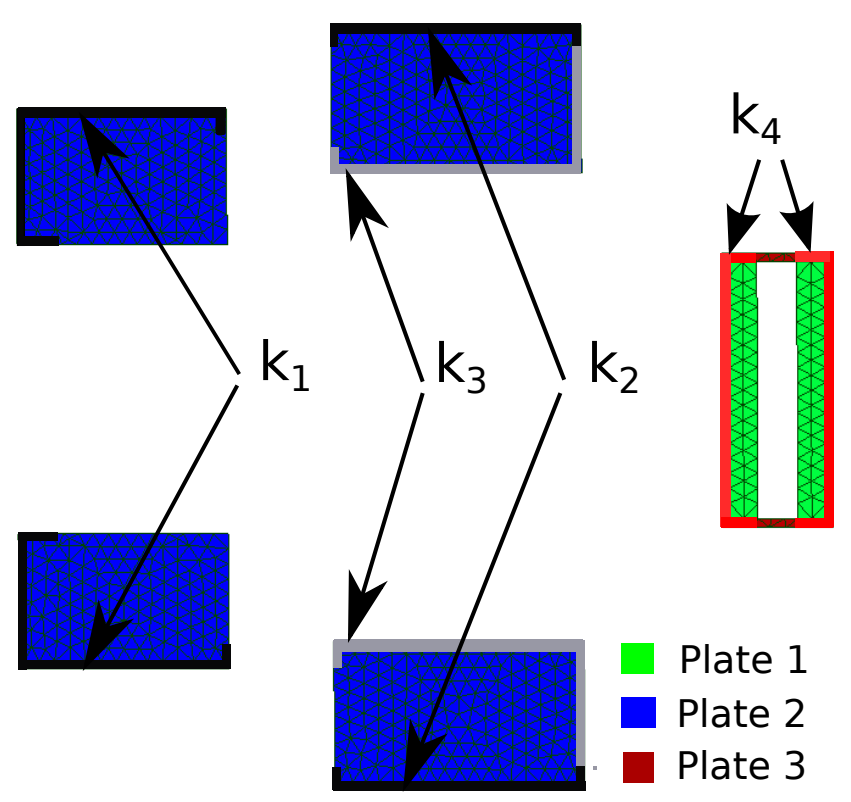

Figure 6: Metallic frame (bottom view).

the updated NCM for these two pumps are different. The differences between the two updated NCM are relative to the values of some (but not all) parameters that have been chosen as updating parameters. The values of the parameters for the updated NCM related to the two pumps are reported in Table 1. An infinite for a discrete spring means that this discrete spring has been replaced by a direct coupling.

The uncertain model parameters are the eight parameters which have been updated. Let $\mathbf{h}=\left(y_{s}, y_{c}, t_{1}, t_{2}, t_{3}, k_{1}, k_{2}, k_{3}, k_{4}\right)$ be the vector of these uncertain model parameters. For the updated NCM of Pump 1 and Pump 2, the updated values of $\mathbf{h}$ are denoted by $\mathbf{h}^{1}$ and $\mathbf{h}^{2}$. For each updated NCM, $n=20$ modes are calculated . For $\mathbf{h}=\mathbf{h}^{1}$, the first 6 eigenfrequencies are $76.4 \mathrm{~Hz}, 81.2 \mathrm{~Hz}, 143.1 \mathrm{~Hz}, 163.5 \mathrm{~Hz}, 185.5 \mathrm{~Hz}, 200.1 \mathrm{~Hz}$ and the first two mode shapes $\phi_{1}\left(\mathbf{h}^{1}\right)$ and $\phi_{2}\left(\mathbf{h}^{1}\right)$ are plotted in Fig. 7 . For Pump 1, the first 6 projected mode shapes are plotted in Fig. 8. For Pump 1, the MAC matrix 


\begin{tabular}{|l|c|c|}
\hline & Pump 1 & Pump 2 \\
\hline Young's modulus steel $y_{s}(\mathrm{~Pa})$ & $1.43 \times 10^{11}$ & $1.40 \times 10^{11}$ \\
\hline Young's modulus cast iron $y_{c}(\mathrm{~Pa})$ & $8.2 \times 10^{10}$ & $8.0 \times 10^{10}$ \\
\hline Thickness $t_{1}(\mathrm{~m})$ & 0.024 & 0.024 \\
\hline Thickness $t_{2}(\mathrm{~m})$ & 0.015 & 0.015 \\
\hline Thickness $t_{3}(\mathrm{~m})$ & 0.017 & 0.017 \\
\hline Stiffness $k_{1}(\mathrm{~N} / \mathrm{m})$ & $\infty$ & $1.0 \times 10^{9}$ \\
\hline Stiffness $k_{2}(\mathrm{~N} / \mathrm{m})$ & $\infty$ & $1.0 \times 10^{10}$ \\
\hline Stiffness $k_{3}(\mathrm{~N} / \mathrm{m})$ & $1.0 \times 10^{8}$ & $\infty$ \\
\hline Stiffness $k_{4}(\mathrm{~N} / \mathrm{m})$ & $3.0 \times 10^{8}$ & $1.0 \times 10^{8}$ \\
\hline
\end{tabular}

Table 1: Values of the parameters for updated NCM of Pump 1 and Pump 2.
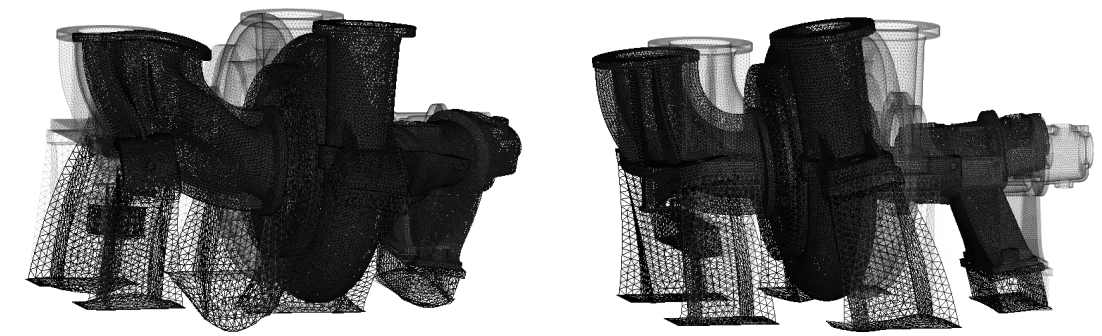

Figure 7: Mode shapes $\phi_{1}\left(\mathbf{h}^{1}\right)$ and $\phi_{2}\left(\mathbf{h}^{1}\right)$ for Pump 1.
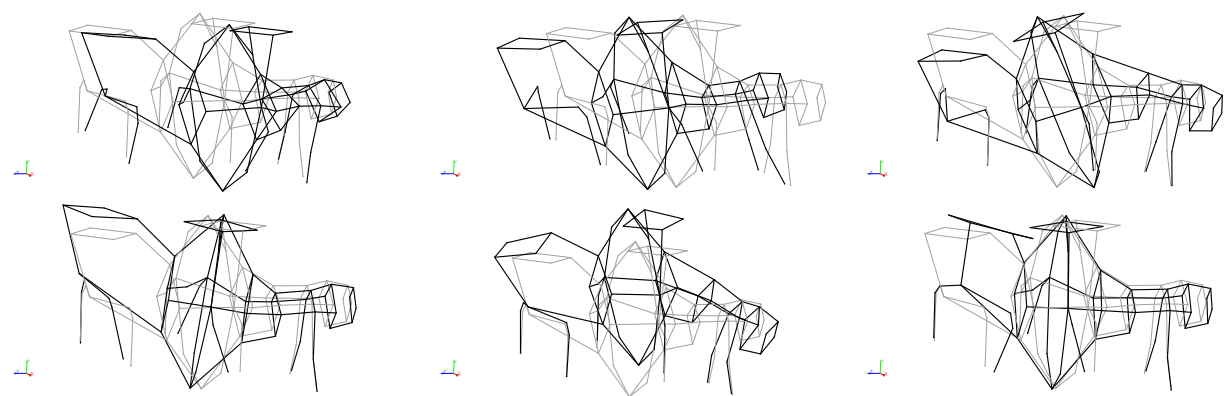

Figure 8: First 6 projected mode shapes for Pump 1.

between the projected computed mode shapes of the updated NCM and the experimental mode shapes is plotted in Fig. 9. For $\mathbf{h}=\mathbf{h}^{2}$, the first 6 eigen- 


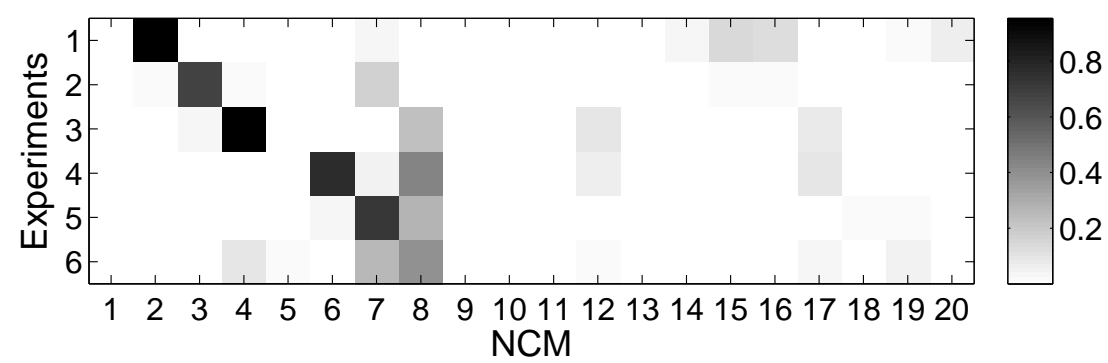

Figure 9: MAC matrix for the updated NCM of Pump 1.

frequencies are $78.6 \mathrm{~Hz}, 82.4 \mathrm{~Hz}, 140.7 \mathrm{~Hz}, 157.4 \mathrm{~Hz}, 181.6 \mathrm{~Hz}, 192.9 \mathrm{~Hz}$ and the first 6 projected mode shapes are plotted in Fig. 10. For Pump 2, the
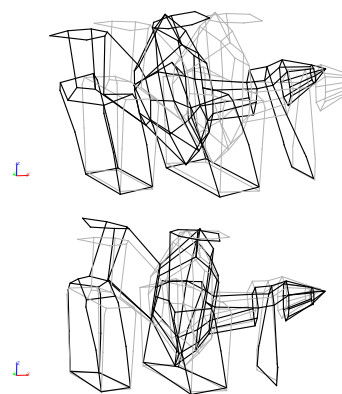
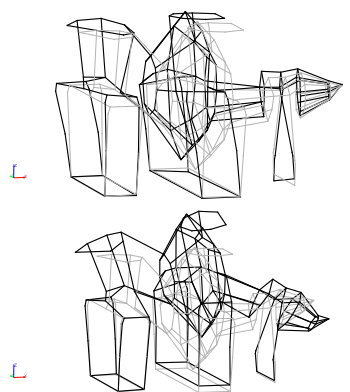
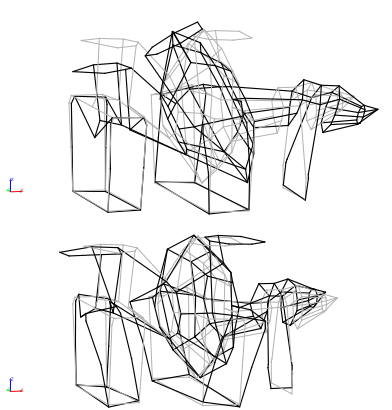

Figure 10: First 6 projected mode shapes for Pump 2.

MAC matrix between the projected computation mode shapes of the updated NCM and the experimental mode shapes is plotted in Fig. 11. It can be seen for Pump 1 and Pump 2 that the MAC indices are low. Therefore, there is no clear correspondence between the experimental modes and the modes calculated with the updated NCM. The transformed modal bases $\left[\widehat{\Phi}^{1}\left(\mathbf{h}^{1}\right)\right]$ and $\left[\widehat{\Phi}^{2}\left(\mathbf{h}^{2}\right)\right]$ are calculated using Eq. (21). The MAC matrix between the experimental modal basis $\left[\widehat{\boldsymbol{\Phi}}^{\exp , 1}\right]$ and the transformed computational modal basis $\left[\widehat{\Phi}^{1}\left(\mathbf{h}^{1}\right)\right]$ is plotted in Fig. 12 and the MAC matrix between the experimental modal basis $\left[\widehat{\boldsymbol{\Phi}}^{\exp , 2}\right]$ and the transformed computational modal basis $\left[\widehat{\Phi}^{2}\left(\mathbf{h}^{2}\right)\right]$ is plotted in Fig. 13. In Figs. 12 and 13, it can be seen that the new MAC matrix between the transformed modal bases of the updated NCM and the experimental modal bases are good. 


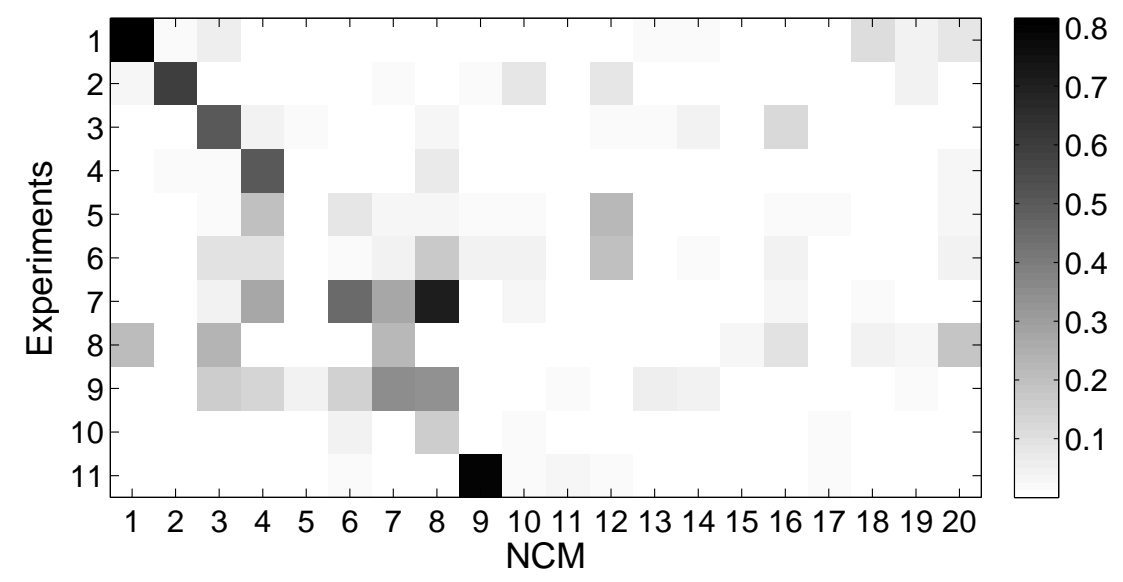

Figure 11: MAC matrix for the updated NCM of Pump 2.

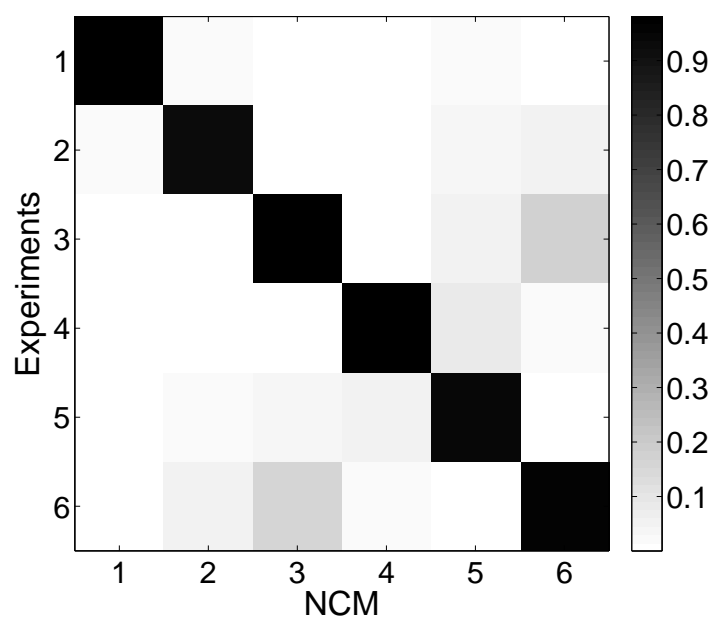

Figure 12: MAC matrix for the updated NCM of Pump 1 after transformation of the mode shapes.

\subsubsection{Construction of the stochastic computational model}

The vector $\mathbf{h}$ is modeled by a random vector $\mathbf{H}=\left(Y_{s}, Y_{c}, T_{1}, T_{2}, T_{3}, K_{1}, K_{2}\right.$, $\left.K_{3}, K_{4}\right)$. The Maximum Entropy principle has been used for constructing the probability distribution of $\mathbf{H}$. Taking into account the available information, it can be deduced that (1) all the components of $\mathbf{H}$ are independent random 


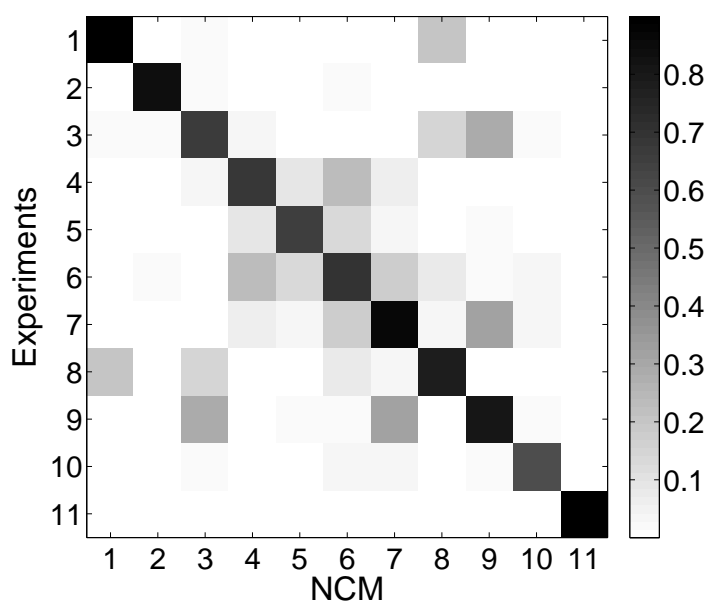

Figure 13: MAC matrix for the updated NCM of Pump 2 after transformation of the mode shapes.

variables; (2) positive-valued Young moduli $Y_{s}$ and $Y_{c}$ are Gamma random variables parameterized by their mean values $m_{Y_{s}}$ and $m_{Y_{c}}$, and by their coefficients of variation (standard deviation divided by the mean value) $\delta_{Y_{s}}$ and $\delta_{Y_{c}} ;(3)$ positive-valued random thicknesses $T_{1}, T_{2}$ and $T_{3}$ are uniform positive-valued random variables parameterized by their mean values $m_{T_{1}}$, $m_{T_{2}}$ and $m_{T_{3}}$, and by their coefficients of variation $\delta_{T_{1}}, \delta_{T_{2}}$ and $\delta_{T_{3}} ;$; (4) positive-valued stiffnesses $K_{1}, K_{2}, K_{3}$ and $K_{4}$ are Gamma random variables parameterized by their mean values $m_{K_{1}}, m_{K_{2}}, m_{K_{3}}$ and $m_{K_{4}}$, and by their coefficients of variation $\delta_{K_{1}}, \delta_{K_{2}}, \delta_{K_{3}}$ and $\delta_{K_{4}}$. Then $\boldsymbol{\alpha}_{\mathrm{par}}=\left(m_{Y_{s}}, \delta_{Y_{s}}, m_{Y_{c}}\right.$, $\left.\delta_{Y_{s}}, m_{T_{1}}, \delta_{T_{1}}, m_{T_{2}}, \delta_{T_{2}}, m_{T_{3}}, \delta_{T_{3}}, m_{K_{1}}, \delta_{K_{1}}, m_{K_{2}}, \delta_{K_{2}}, m_{K_{3}}, \delta_{K_{3}}, m_{K_{4}}, \delta_{K_{4}}\right)$ has 18 components to be identified and $\boldsymbol{\alpha}=\left(\boldsymbol{\alpha}_{\text {par }}, \boldsymbol{\alpha}_{\text {mod }}\right)$ has 20 components to be identified.

\subsubsection{Identification of the optimal hyperparameter $\boldsymbol{\alpha}^{\text {opt }}$.}

The vector $\boldsymbol{\alpha}^{\mathrm{opt}}$ is given by the optimization problem defined by Eq. (28) which is solved using a genetic algorithm. For each value of $\boldsymbol{\alpha}$, the probability density functions $p_{\mathbf{w}^{1}}(\mathbf{w} ; \boldsymbol{\alpha})$ and $p_{\mathbf{w}^{2}}(\mathbf{w} ; \boldsymbol{\alpha})$ are estimated using 800 realizations of the observation vectors $\mathbf{W}^{1}$ and $\mathbf{W}^{2}$ calculated with the SCM. The components of $\boldsymbol{\alpha}_{\mathrm{par}}^{\mathrm{opt}}$ are given in Table 2. The two components of $\boldsymbol{\alpha}_{\mathrm{mod}}^{\mathrm{opt}}$ are $\delta_{M}^{\mathrm{opt}}=0.52$ and $\delta_{K}^{\mathrm{opt}}=0.43$. These optimal dispersions of the probability distributions for model uncertainties are relatively high due to the experi- 
mental variability and due to modeling errors introduced in the NCM. These dispersions could be partly decreased by constructing a more accurate NCM.

\begin{tabular}{|l|c|c|}
\hline & Mean value & Coefficient of variation \\
\hline Young's modulus steel $Y_{s}$ & $m_{Y_{s}}=1.33 \times 10^{11} \mathrm{~Pa}$ & $\delta_{Y_{s}}=0.2$ \\
\hline Young's modulus cast iron $Y_{c}$ & $m_{Y_{c}}=7.57 \times 10^{10} \mathrm{~Pa}$ & $\delta_{Y_{c}}=0.17$ \\
\hline Thickness $T_{1}$ & $m_{T_{1}}=0.013 \mathrm{~m}$ & $\delta_{T_{1}}=0.12$ \\
\hline Thickness $T_{2}$ & $m_{T_{2}}=0.011 \mathrm{~m}$ & $\delta_{T_{2}}=0.57$ \\
\hline Thickness $T_{3}$ & $m_{T_{3}}=0.01 \mathrm{~m}$ & $\delta_{T_{3}}=0.09$ \\
\hline Stiffness $K_{1}$ & $m_{K_{1}}=8.01 \times 10^{8} \mathrm{~N} / \mathrm{m}$ & $\delta_{K_{1}}=0.19$ \\
\hline Stiffness $K_{2}$ & $m_{K_{2}}=5.74 \times 10^{9} \mathrm{~N} / \mathrm{m}$ & $\delta_{K_{2}}=0.13$ \\
\hline Stiffness $K_{3}$ & $m_{K_{3}}=6.30 \times 10^{7} \mathrm{~N} / \mathrm{m}$ & $\delta_{K_{3}}=0.34$ \\
\hline Stiffness $K_{4}$ & $m_{K_{4}}=2.68 \times 10^{8} \mathrm{~N} / \mathrm{m}$ & $\delta_{K_{4}}=0.42$ \\
\hline
\end{tabular}

Table 2: Components of $\boldsymbol{\alpha}_{\text {par }}^{\mathrm{opt}}$.

\subsection{Validation of the results}

For $\boldsymbol{\alpha}=\boldsymbol{\alpha}^{\mathrm{opt}}$, the marginal pdf of the first six eigenvalues of the matrices $\left[\widehat{\mathbf{K}}^{1}\right]$ and $\left[\widehat{\mathbf{K}}^{2}\right]$ are shown in Figs. 14 and 15 respectively. It can be seen in these figures that the first 5 experimental eigenvalues for Pump 1 and Pump 2 are predicted by the SCM with a high probability level (very high for Pump 1). The $6^{\text {th }}$ experimental eigenvalue for Pump 1 and Pump 2 are also within the interval of prediction of the SCM but with a lower probability level.

For Pump 1, the mean value of the MAC matrix between the random modal basis $\left[\widetilde{\boldsymbol{\Phi}}^{1}\right]$ (before transformation) of the SCM and the experimental modal basis $\left[\widehat{\Phi}^{\text {exp,1}}\right]$ is plotted in Fig. 16, while for Pump 2, the mean value of MAC matrix is plotted in Fig. 17. The comparison of Figs. 16 and 17 with Figs. 9 and 11 shows that the randomness of the SCM introduces random mode crossings and random mode veerings which modify the correspondence. For Pump 1, the mean value of the MAC matrix between the random modal basis $\left[\widehat{\boldsymbol{\Phi}}^{1}\right]$ (after transformation) of the SCM and the experimental modal basis $\left[\widehat{\Phi}^{\text {exp,1}}\right]$ is plotted in Fig. 18 while for Pump 2, the mean value of MAC matrix is plotted in Fig. 19. In Figs. 18 and 19, it can be seen that the random transformation of the random mode shapes of the SCM allows to 
achieve a good correspondence between the random computational modes of the SCM and the experimental modes.
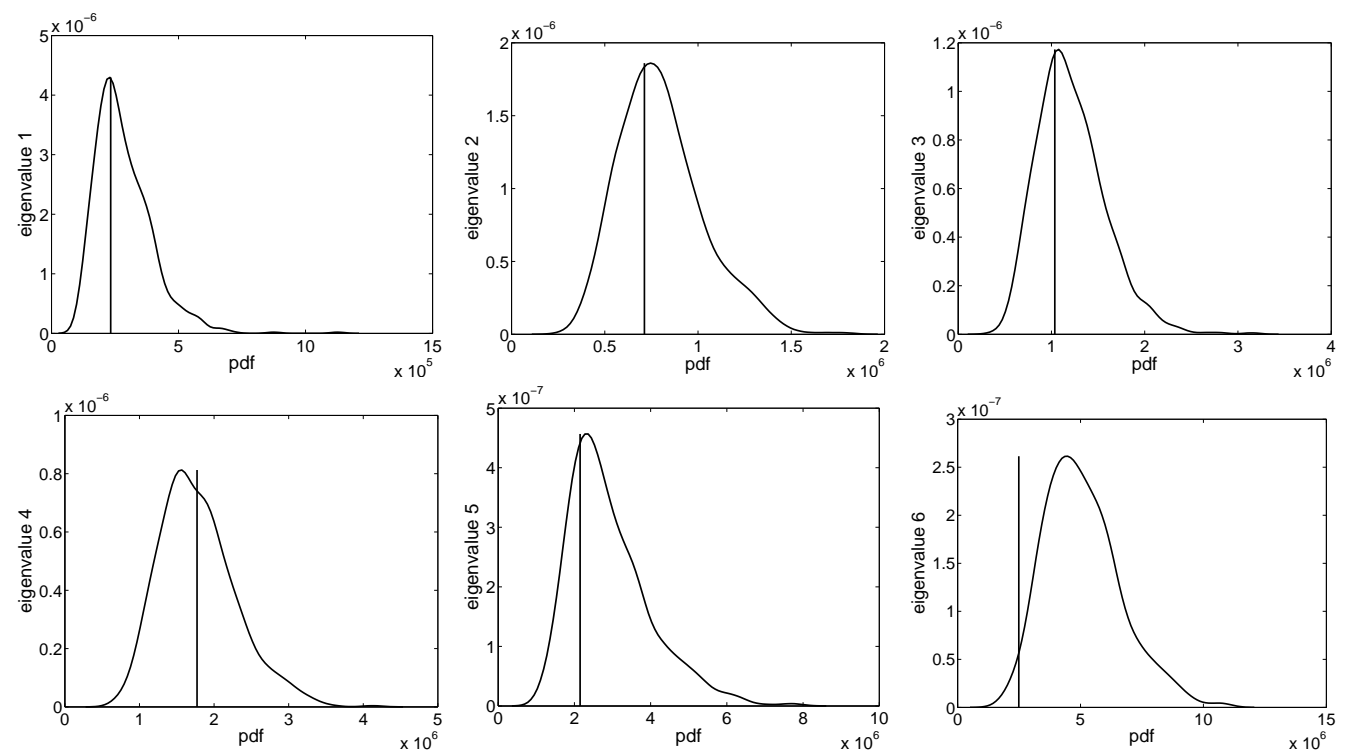

Figure 14: Probability density function for the first six eigenvalues of $\left[\widehat{\mathbf{K}}^{1}\right]$. Vertical lines: corresponding experimental values (eigenvalues of $\left[\widehat{K}^{\exp , 1}\right]$ ).

\section{Conclusion}

A new methodology has been presented for the identification of a stochastic computational model using experimental eigenfrequencies and mode shapes with experimental variabilities. The model-parameter uncertainties and the modeling errors are taken into account in the framework of a generalized probabilistic approach of uncertainties. A transformation of the computational modal quantities is introduced in order to construct a correspondence between the experimental modal data and the computational modal quantities. This method allows us to take into account mode crossings and mode veerings that may occur. The methodology has been applied to the construction a stochastic computational model representing a family of booster pumps of thermal units. The first 5 experimental eigenvalues for Pump 1 and Pump 2 are predicted by the SCM with a high probability level. 

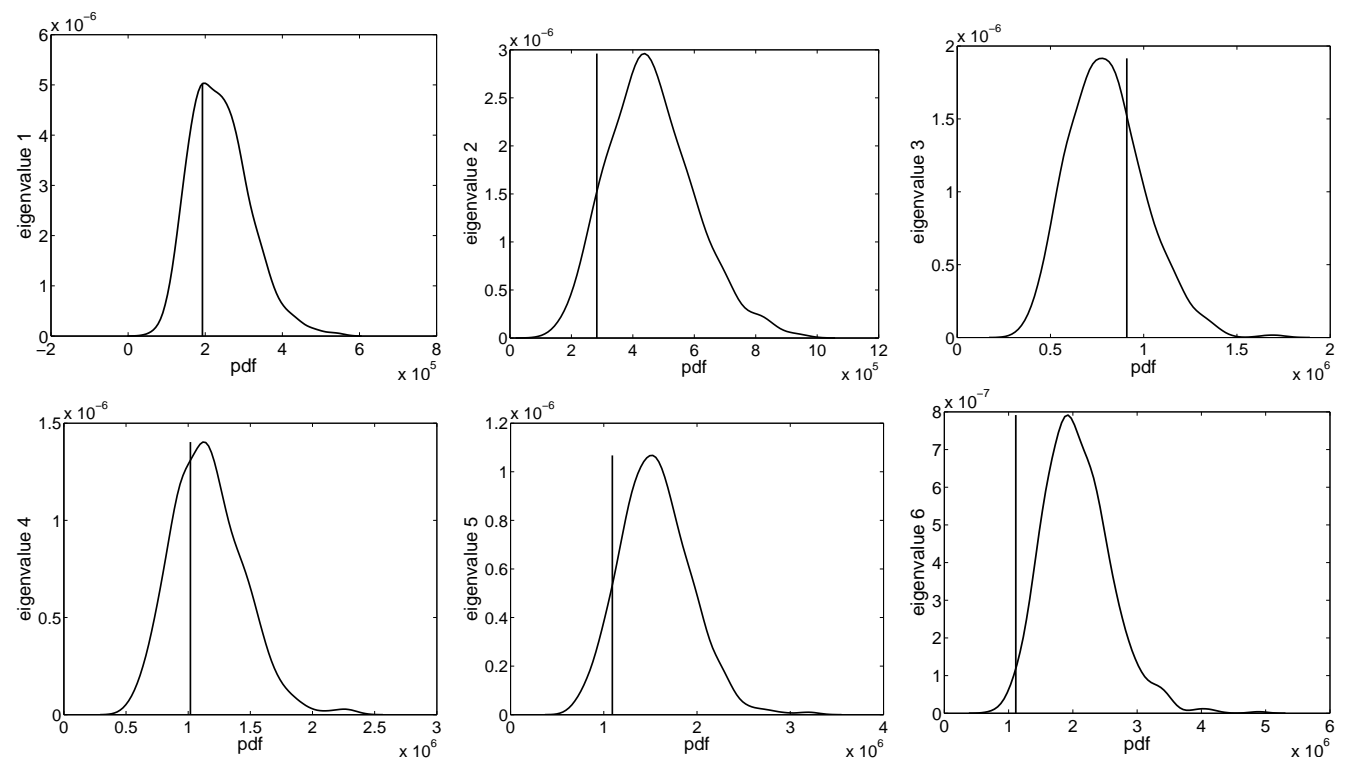

Figure 15: Probability density function for the first six eigenvalues of $\left[\widehat{\mathbf{K}}^{2}\right]$. Vertical lines: corresponding experimental values (eigenvalues of $\left[\widehat{K}^{\exp , 2}\right]$ ).

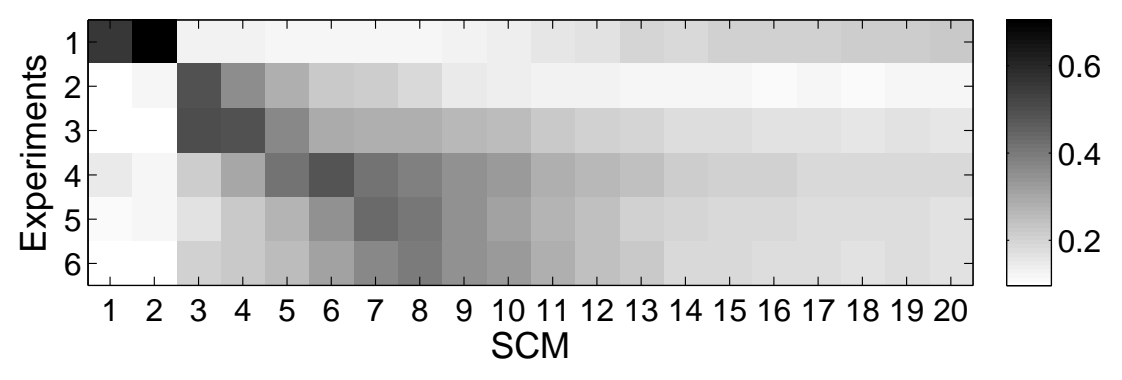

Figure 16: For Pump 1, mean value of the MAC matrix between the random mode shapes of the SCM and the experimental mode shapes before transformation.

The dispersion of the probability distributions for model uncertainties that have been identified are relatively high due to the experimental variability and due to modeling errors introduced in the NCM. These dispersions could be partly decreased by constructing a more accurate NCM with a better representation of the bolted joints and the boundary conditions. The experimental variabilities induced by the measurements errors have not 


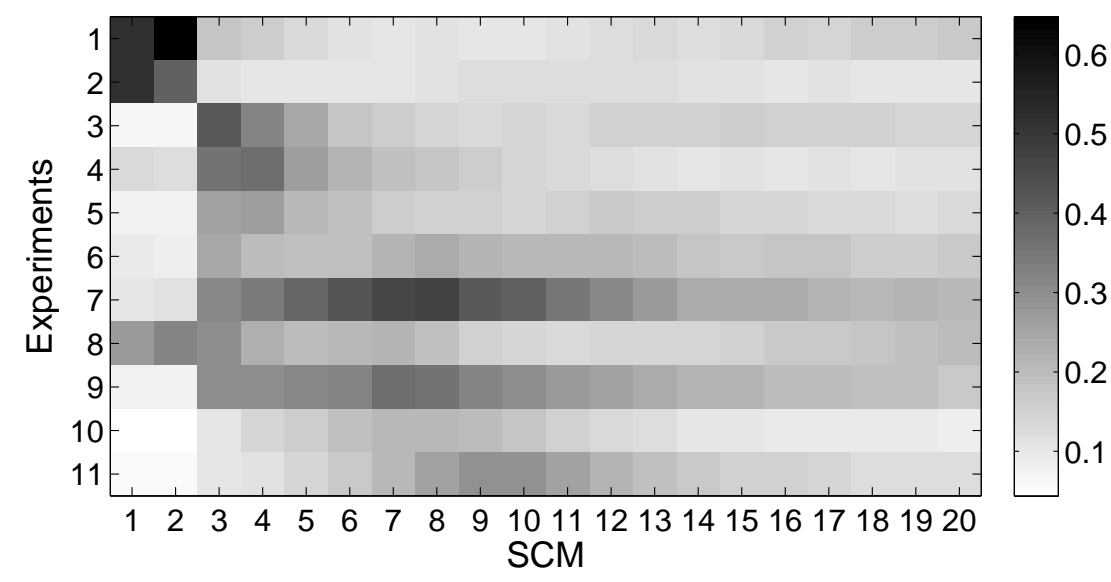

Figure 17: For Pump 2, mean value of the MAC matrix between the random mode shapes of the SCM and the experimental mode shapes before transformation.

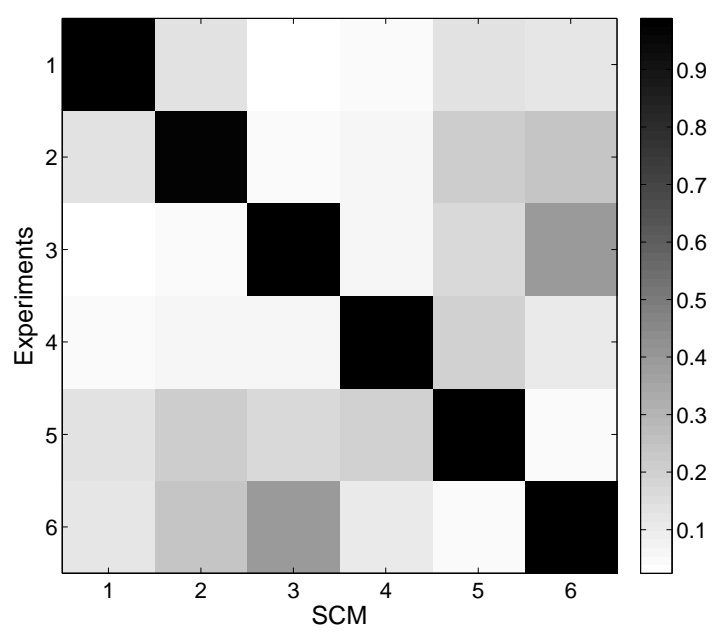

Figure 18: For Pump 1, mean value of the MAC matrix between the random mode shapes of the SCM and the experimental mode shapes after transformation.

directly been modeled, but are indirectly taken into account by the probabilistic model of model uncertainties. Therefore, in the present paper, this experimental uncertainty is not quantified independently but is encompassed within the model uncertainties which have been quantified. 


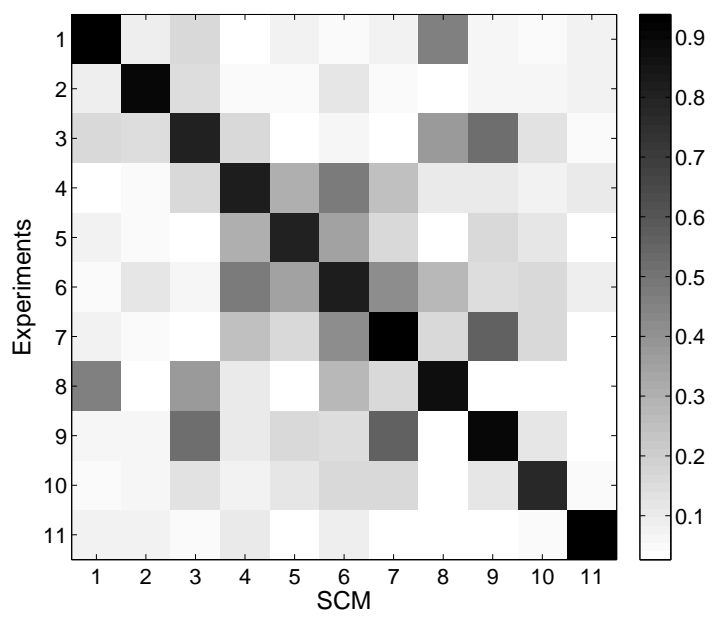

Figure 19: For Pump 2, mean value of the MAC matrix between the random mode shapes of the SCM and the experimental mode shapes after transformation.

The introduction of a proper stochastic model of measurement errors which could be done by introducing additive random noises could also partly decrease the values of the dispersions of model uncertainties which have been identified, but in counter part, a stochastic model of such additive random noises must be constructed and must experimentally be identified.

\section{Acknowledgements}

This research work has been carried out in the context of the FUI 20122015 SICODYN Project (pour des SImulations crédibles via la COrrélation calculs-essais et l'estimation des incertitudes en DYNamique des structures). The support of the FUI (Fonds Unique Interministériel) is gratefully acknowledged.

\section{References}

[1] D. Amsallem, C. Farhat, An online method for interpolating linear parametric reduced-order models, SIAM Journal on Scientific Computing. 33(5) (2011) 2169-2198. 
[2] J. Avalos, E. D. Swenson, M. P. Mignolet, N. J. Lindsley, Stochastic modeling of structural uncertainty/variability from ground vibration modal test data, Journal of Aircraft. 49(3) (2012) 870-884.

[3] M. Baruch, Optimisation procedure to correct stiffness and flexibility matrices using vibration data, AIAA Journal. 16(11) (1978) 1208-1210.

[4] M. Baruch, Modal data are insufficient for identification of both mass and stiffness matrices, AIAA journal. 35(11) (1997) 1797-1798.

[5] A. Batou, C., Soize, M. Corus, Experimental identification of an uncertain computational dynamical model representing a family of structures, Computer and Structures. 89(13-14) (2011) 1440-1448.

[6] Y. Ben-Haim, U. Prells, Selective sensitivity in the frequency domain: Theory, Mechanical Systems and Signal Processing. 7 (1993) 461-475.

[7] J.M.F.T. Berge, D. L. Knol, Orthogonal rotations to maximal agreement for two or more matrices of different column orders, Psychometrika. 49(1) (1984) 49-55.

[8] A. Bojanczyk, A. Lutoborski, The procrustes problem for orthogonal Stiefel matrices, SIAM Journal on Scientific Computing. 21(4) (1999) 1291-1304.

[9] A. T. Bollinger, Finite element model updating for FEA/EMA modal correlation via constrained optimization theory, IMAC Proc., 882-888, 1994.

[10] R. Brincker, A. Skafte, M. López-Aenlle, A. Sestieri, W. D'Ambrogio, A. Canteli, A local correspondence principle for mode shapes in structural dynamics , Mechanical Systems and Signal Processing. 45(1) (2014) 91104.

[11] O. Brüls, P. Duysinx, J. C. Golinval, The global modal parameterization for non-linear model-order reduction in flexible multibody dynamics, Internat. J. Numer. Methods Engrg. 69 (2007) 958-977.

[12] E. Capiez-Lernout, C. Soize, Robust updating of uncertain damping models in structural dynamics for low- and medium-frequency ranges, Mechanical Systems and Signal Processing. 22(8) (2008) 1774-1792. 
[13] S. H. Cheung, J. L. Beck, Bayesian model updating using hybrid Monte Carlo simulation with application to structural dynamic models with many uncertain parameters, Journal of Engineering Mechanics-ASCE. 135(4) (2009), 243-255.

[14] S. Cogan, D. Lenoir, G. Lallement, An improved frequency response residual for model correction, IMAC Proc., 568-573, 1995.

[15] S. Cogan, G. Lallement, F. Ayer, Y. Ben-Haim, Model order reduction by selective sensitivity, AIAA Journal. 35(3) (1997) 557-562.

[16] A. Edelman, T. A. Arias, S. T. Smith, The geometry of algorithms with orthogonality constraints, SIAM J. Matrix Anal. Appl. 20(2) (1998), 303-353.

[17] A. Eiben, J. Smith, Introduction to Evolutionary Computing, Springer, 2003.

[18] D. J. Ewins, Modal Testing: Theory and Practice, Research Study Press LTD, John Wiley and Sons, 1984.

[19] B. Faverjon, P. Ladevèze, F. Louf, An updating method for structural dynamics models with uncertainties, Shock and Vibration. 15(3-4) (2008) 245-256, 2008.

[20] M. I. Friswell, J. E. Mottershead, Finite Element Model Updating in Structural Dynamics, Kluwer Academic Publishers, 1995.

[21] M. I. Friswell, D. J. Inman, D. F. Pilkey, Direct updating of damping and stiffness matrices, AIAA journal. 36(3) (1998), 491-493.

[22] B. Goller, H. J. Pradlwarter, G. I. Schueller, Robust model updating with insufficient data, Computer Methods in Applied Mechanics and Engineering. 198(37-40) (2009), 3096-3104.

[23] B. Goller, J. L. Beck, G. I. Schueller, Evidence-based identification of weighting factors in Bayesian model updating using modal data, Journal of Engineering Mechanics-ASCE. 138(5) (2012), 430-440.

[24] G. H. Golub, C. F. Loan, Matrix Computations, $2^{\text {nd }}$ ed., The John Hopkins University Press, Baltimor, 1989. 
[25] E. T. Jaynes, Information theory and statistical mechanics, Phys. Rev. 106(4) (1957) 620-630 and 108(3) (1957) 171-190.

[26] J. N. Kapur, H. K. Kesavan, Entropy Optimization Principles with Applications, Academic Press, San Diego, 1992.

[27] R. Kenigsbuch, Y. Halevi, Model updating in structural dynamics: A generalised reference basis approach, Mechanical Systems and Signal Processing. 12(1) (1998) 75-90.

[28] T. S. Kim, Y. Y. Kim, Mac-based mode tracking in structural topology optimization, Computers \& Structures. 74(3) (2000) 375-383.

[29] L. W. Levine-West, M. Milman, A. Kissil, Mode shape expansion techniques for prediction - Experimental evaluation, AIAA Journal. 34(4) (1996) 821-829.

[30] R. I. Levin, N. A. J. Lieven, Dynamic finite element model updating using simulated annealing and genetic algorithms, Mechanical Systems and Signal Processing. 12(1) (1998) 91-120.

[31] L. Ljung, System Identification: Theory for the User, Prentice-Hall, New York, 1987.

[32] K. McConnel, Vibration Testing. Theory and Practice, Wiley Interscience, New-York, 1995.

[33] H.J.-P. Morand, R. Ohayon, Fluid-Structure Interaction, Wiley, New York, 1995.

[34] C. Mares, J. Mottershead, M. Friswell, Stochastic model updating, Part 1: Theory and simulated example, Mechanical Systems and Signal Processing. 20(7) (2006) 1674-1695.

[35] J. Mottershead, C. Mares, S. James, M. Friswell, Stochastic model updating, Part 2: Application to a set of physical structures, Mechanical Systems and Signal Processing. 20(8) (2006) 2171-2185.

[36] N. C. Perkins, C. D. Mote Jr, Comments on a curve veering in eigenvalue problems, Journal of Sound and Vibration. 106(3) (1986) 451-463. 
[37] C. Pierre, Mode localization and eigenvalue loci veering phenomena in disordered structures, Journal of Sound and Vibration. 126(3) (1988) $485-502$.

[38] R. Y. Rubinstein, D. P. Kroese, Simulation and the Monte Carlo Method, 2nd edn, John Wiley and Sons, New York, 2008.

[39] G. I Schueller, A state-of-the-art report on computational stochastic mechanics, Probabilistic Engineering Mechanics, 12(4) (1997) 197-313.

[40] G. I Schueller, H. J. Pradlwarter, Uncertain linear systems in dynamics: Retrospective and recent developments by stochastic approaches, Engineering Structures. 31(11) (2009) 2507-2517.

[41] M. J. Schultz, D. J. Inman, Model updating using constrained eigenstructure assignment, Journal of Sound and Vibration. 178(1) (1994) 113-130.

[42] C. E. Shannon, A mathematical theory of communication, Bell System Technology Journal. 27 (1948) 379-423 and 623-659.

[43] S. W. Smith, C. A. Beattie, Simultaneous expansion and orthogonalization of measured modes for structure identification, Proc. AIAA Dynamics Specialist Conference, Long Beach, Ca., April 1990.

[44] C. Soize, A nonparametric model of random uncertainties for reduced matrix models in structural dynamics, Probabilistic Engineering Mechanics. 15(3) (2000) 277-294.

[45] C. Soize, E. Capiez-Lernout, R. Ohayon, Robust updating of uncertain computational models using experimental modal analysis, AIAA Journal. 46(11) (2008) 2955-2965.

[46] C. Soize, E. Capiez-Lernout, J.-F. Durand, C. Fernandez, L. Gagliardini, Probabilistic model identification of uncertainties in computational models for dynamical systems and experimental validation, Computer Methods in Applied Mechanics and Engineering. 198(1) (2008) 150-163.

[47] C. Soize, Generalized probabilistic approach of uncertainties in computational dynamics using random matrices and polynomial chaos decomposition, International Journal for Numerical Methods in Engineering. 81(8) (2010) 939-970. 
[48] C. Soize, Stochastic modeling of uncertainties in computational structural dynamics - Recent theoretical advances, Journal of Sound and Vibration. 332(10) (2013) 2379-2395.

[49] C. Soize, Bayesian posteriors of uncertainty quantification in computational structural dynamics for low- and medium-frequency ranges, Computers and Structures. 126 (2013) 41-55.

[50] J. C. Spall, Introduction to Stochastic Search and Optimization, John Wiley and Sons, Hoboken, New Jersey, 2003.

[51] E. Walter, L. Pronzato, Identification of Parametric Models from Experimental Data, Springer-Verlag, Berlin, 1997.

[52] M. P. Wand, M. C. Jones, Kernel Smoothing, Chapman \& Hall, London, U.K., 1995.

[53] K. V. Yuen, Updating large models for mechanical systems using incomplete modal measurement, Mechanical Systems and Signal Processing. 28 (2012) 297-308.

[54] D. C. Zimmerman, T. Simmermacher, Model correlation using multiple static load and vibration tests, AIAA journal. 33(11) (1995) 2182-2188. 